Clemson University

TigerPrints

August 2021

\title{
Shining Light on Retroreflectivity: Perceived Brightness of Surfaces with Varying Reflective Properties
}

Savana King

Clemson University, savanalking@gmail.com

Follow this and additional works at: https://tigerprints.clemson.edu/all_theses

\section{Recommended Citation}

King, Savana, "Shining Light on Retroreflectivity: Perceived Brightness of Surfaces with Varying Reflective Properties" (2021). All Theses. 3607.

https://tigerprints.clemson.edu/all_theses/3607

This Thesis is brought to you for free and open access by the Theses at TigerPrints. It has been accepted for inclusion in All Theses by an authorized administrator of TigerPrints. For more information, please contact kokeefe@clemson.edu. 
SHINING LIGHT ON RETROREFLECTIVITY: PERCEIVED BRIGHTNESS OF
SURFACES WITH VARYING REFLECTIVE PROPERTIES

\begin{tabular}{c} 
A Thesis \\
Presented to \\
the Graduate School of \\
Clemson University \\
\hline In Partial Fulfillment \\
of the Requirements for the Degree \\
Master of Science \\
Applied Psychology \\
\hline Savana Laine King \\
August 2021 \\
br. Richard Tyrrell, Committee Chair \\
Dr. Benjamin Stephens \\
Dr. Zhuo Job Chen \\
Accepted by:
\end{tabular}




\begin{abstract}
Typical road users appear not to understand retroreflectivity despite nightly exposure to retroreflective materials such as road signs. While retroreflective surfaces have been engineered to reflect light back in the direction of its source, retroreflectors appear to be diffuse reflectors in most viewing conditions. This may lead road users to underestimate its value in nighttime roadway applications and contribute to vulnerable road users' under-use of retroreflective markings to enhance their own conspicuity at night. In this context, a critical benefit of retroreflective materials is a robustness to changes in entrance angle, the angle at which the light strikes the material.

This online study measured participants' perceived brightness judgments for surfaces that represent three types of reflection (diffuse, specular, and retroreflective) with varying entrance angles. To assess the potential benefits of seeing retroreflectivity in action, perceived brightness was evaluated before and during a demonstration that includes illumination from a directional light source positioned near the recording camera. After seeing the visual demonstrations, participants' brightness judgments revealed an enhanced appreciation that retroreflective (but not specular or diffuse) surfaces remain bright despite large changes in entrance angle. These findings could be utilized to design more effective and accessible video demonstrations of retroreflectivity in hopes of increasing the demand for retroreflective markings by vulnerable road users.
\end{abstract}




\section{ACKNOWLEDGMENTS}

I would like to thank my advisor, Dr. Rick Tyrrell, for his plentiful insight and encouragement. I also greatly appreciate the expertise of my committee members Dr. Ben Stephens and Dr. Job Chen. Lastly, I am grateful to my husband, Logan, for his unending positivity when I just needed a hug. The guidance and perspective of each of you was not only deeply of value to me, but also paramount to the success of this project. 


\section{TABLE OF CONTENTS}

Page

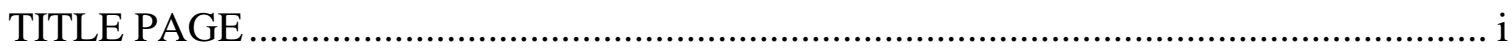

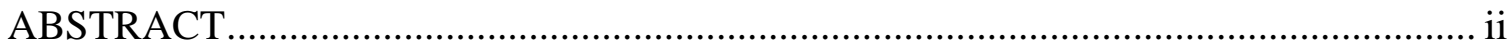

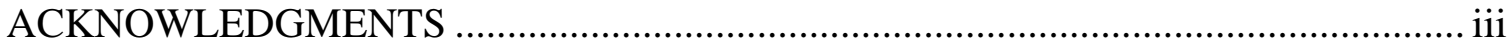

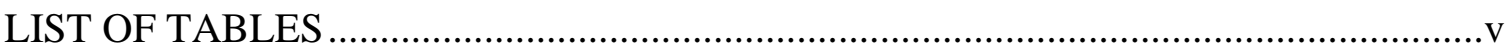

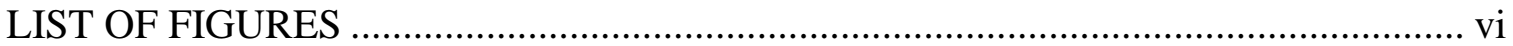

\section{CHAPTER}

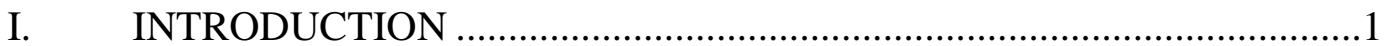

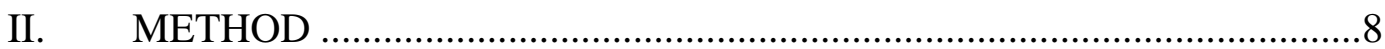

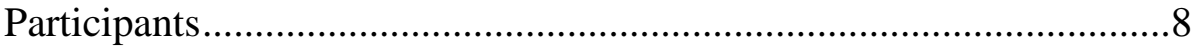

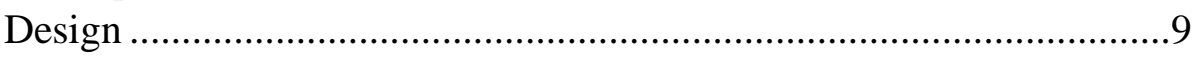

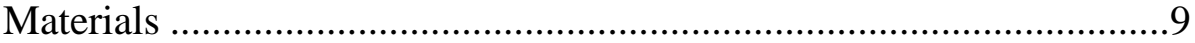

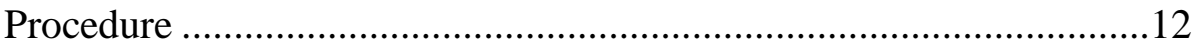

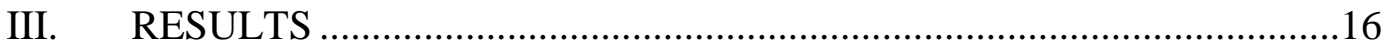

Magnitude Estimation Task ........................................................... 17

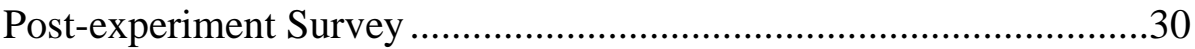

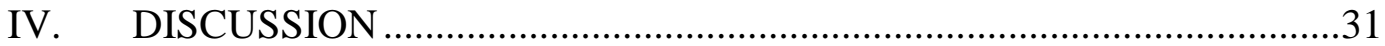

\section{APPENDICES}

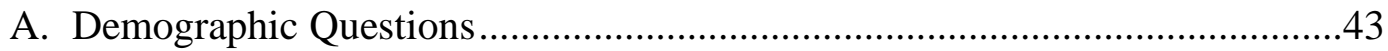

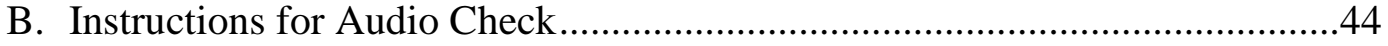

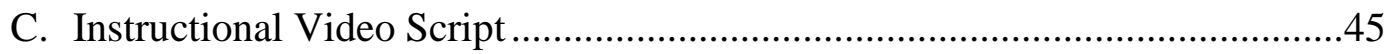

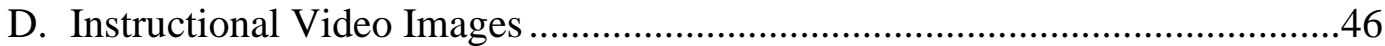

E. Task Comprehension Questions ..............................................................48

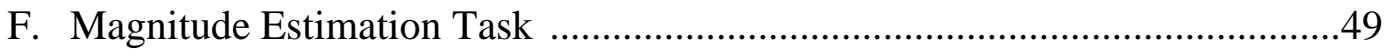

G. Post-experiment Survey Questions ..........................................................50

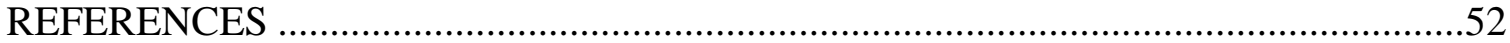




\section{LIST OF TABLES}

Table

Page

1. Magnitude estimate data per condition after geometric averaging procedure ............. 19 


\section{LIST OF FIGURES}

Figure

Page

1. Observation angle and entrance angle as it applies to retroreflectors. Adapted from Federal Highway Administration (2001). Guidelines and Recommendations to Accommodate Older Drivers and Pedestrians (FHWA Publication No. 01-051). U.S.

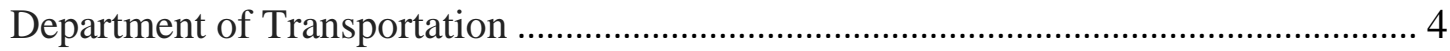

2. Experimental material samples pictured from left to right: diffuse reflective cloth, retroreflective beaded material, and specular reflective mirror

3. Location of LED illuminator during the demonstration phase, in relation to the recording camera

4. The diffuse material sample viewed at each of the three entrance angles, as displayed to participants during the pre-demonstration phase of the experiment

5. Ninety-three participants excluded from analyses, represented by the percent excluded for each reason

6. Geometric mean ( \pm 1 standard error of the mean) brightness predictions during the pre-demonstration phase, as a function of entrance angle and reflectivity type

7. Geometric mean ( \pm 1 standard error of the mean) brightness judgments during the demonstration as a function of entrance angle and reflectivity type

8. The effect of reflectivity type on pre-demonstration brightness predictions and demonstration brightness judgments. These data are averaged across the three entrance angles. Error bars represent \pm 1 standard error of the mean 
9. The effect of entrance angle on pre-demonstration brightness predictions and demonstration brightness judgments, when averaged across reflectivity type. Error bars represent \pm 1 standard error of the mean

10. Specular reflective samples displayed with an entrance angle of 2 degrees, 23 degrees, and 45 degrees

11. Comparison of diffuse (left) and retroreflective (right) samples in the 2-degree, 23degree, and 45-degree conditions during the visual demonstration

12. The effect of reflectivity type on pre-demonstration brightness predictions and demonstration brightness judgments for the 45-degree entrance angle. Error bars represent \pm 1 standard error of the mean

13. Geometric mean brightness for all 18 conditions as a function of presentation phase, reflectivity type, and entrance angle. Error bars represent \pm 1 standard error of the mean

14. Top: Szubski et al.'s magnitude estimation results from an in-person demonstration of reflection with the silver samples affixed to a wall 20 feet $(6 \mathrm{~m})$ from the observers. Bottom: Magnitude estimation results from the current study's online demonstration for the 2-degree condition. Geometric means ( \pm 1 standard error of the mean) are plotted for both studies 


\section{INTRODUCTION}

Pedestrian fatality rates have continued to rise in the United States (Retting, 2020). Disproportionate increases in fatalities from pedestrian-vehicle collisions can be seen when contrasting time of day. Daytime casualties increased by $16 \%$ between 2009 and 2018, compared to the $67 \%$ increase in fatalities at night. Over this nine-year span, a total of 11,873 pedestrians were killed in the US during the day, while 37,298 were killed at night (Retting, 2020). The fact that the number of pedestrian fatalities was more than three times greater at night is particularly alarming given that fewer pedestrian-miles are traveled at night. Tyrrell, Wood, Owens, Whetsel Borzendowski, and Stafford Sewall (2016) emphasized drivers' visual limitations at night and the limited conspicuity of pedestrians at night as a causal factor in the overall rate of pedestrian-vehicle collisions. Road users' lack of awareness of drivers' visual limitations at night can lead to hazardous behaviors including motorists underusing their high beam headlights and pedestrians unknowingly making risky decisions when interacting with traffic at night.

To counter their degraded visibility to drivers at night, pedestrians are urged to consider the conspicuity (or lack thereof) provided by their own clothing. "High visibility" clothing, like vests with built-in fluorescent and retroreflective materials, enhance visibility by accentuating the contrast between themselves and the background against which they are seen. In addition to being visible to motorists, it is important that they are also correctly perceived as pedestrians. As mentioned in Tyrrell et al.'s review article, numerous studies have confirmed that increasing the distance from which drivers recognize the presence of a pedestrian is far less effective when retroreflective markings 
are placed on the torso than when they are placed on the bodily joints (2016). By capitalizing on human's perceptual sensitivity to recognize other humans based on their movements, retroreflective material strategically placed on a pedestrian's knees, ankles, and wrists maximizes their conspicuity in nighttime conditions.

Research has documented that educational interventions can successfully encourage pedestrians to behave in ways that increase their own safety when interacting with traffic at night. These interventions have proven effective in guiding pedestrians to consider high visibility clothing choices (Borzendowski, Sewall, Fekety, \& Tyrrell, 2014) and reduce overestimates of their own nighttime visibility (Tyrrell, Patton, \& Brooks, 2004). The goal of Tyrrell et al.'s research was to explore how effective different educational interventions were at increasing appreciation for the problem of nighttime pedestrian visibility. In two experiments, participants either attended a lecture relevant to the topic, witnessed a demonstration of the nighttime visibility of a pedestrian, or participated in a combination of both instructional methods. Undergraduate students tested within the first experiment were enrolled in an introductory psychology course that included a lecture centered around applied visual physiology and how basic visual science can be applied to enhance safety at night. The second experiment tested high school students enrolled in a driver education course and included a similar lecture. In both studies the demonstration involved participants observing pedestrian experimenters walking (with varying clothing and for both low beam and high beam headlighting) toward and away from the participants' vehicle. To estimate their own visibility, participants walked toward a stationary car until the point they thought they could be just 
recognized as a pedestrian by a person sitting in the car. In both studies participants who had experienced the educational inventions overestimated their own visibility less than those in a control group who had not experienced the interventions. The lecture was particularly effective, resulting in a $56 \%$ reduction in estimated recognition distances compared to the group that did not receive a lecture. Importantly, lecture group estimates within the second experiment tended to vary depending on the clothing and vehicle beam setting. These results were not found within the control group, suggesting that the average pedestrian does not understand the influence that headlight beam settings and retroreflective clothing have on their visibility to drivers of approaching vehicles.

To understand how retroreflection is a powerful tool for increasing the visibility of objects at night, it is worth distinguishing retroreflection from other ways in which light can reflect off surfaces. Specular reflection and diffuse reflection are more commonly experienced in the natural world. Specular "mirrored" reflection occurs when light comes in contact with a microscopically smooth surface. The incident angle is equal to the angle of reflection, permitting the concentrated light that has been reflected to produce a replica of the original image. Still bodies of water, "black" ice, and glass mirrors all demonstrate specular reflectivity. On the other hand, diffuse reflective materials are characterized by dispersing incoming light simultaneously in many directions. Most surfaces that people come in contact with daily (including most clothing fabrics) are microscopically rough and are diffuse reflectors. In the context of the nighttime roadway environment, a key problem with specular and diffuse reflectors is that when headlighting is reflected off an object in the roadway it is not reflected back to 
the driver's eyes. Instead, headlight illumination is reflected farther ahead (in the case of a specular reflector like a patch of black ice) or scattered randomly in many directions (in the case of a diffuse reflector like a cotton t-shirt).

Retroreflective surfaces, on the other hand, reflect incoming light back towards its source. With early examples of commercial grade retroreflection emerging in the 1930s to make road signs more visible at night (Lloyd, 2008), retroreflective materials have been engineered and refined specifically to enhance object visibility to nighttime drivers. The retroreflective markings on a pedestrian, for example, can appear unusually bright and can therefore stand in high contrast relative to the dark background. From the perspective of a driver sitting above a pair of headlamps, the luminance of illuminated retroreflective material is dependent on two important angles: the angle of observation and the angle of entrance (Figure 1).

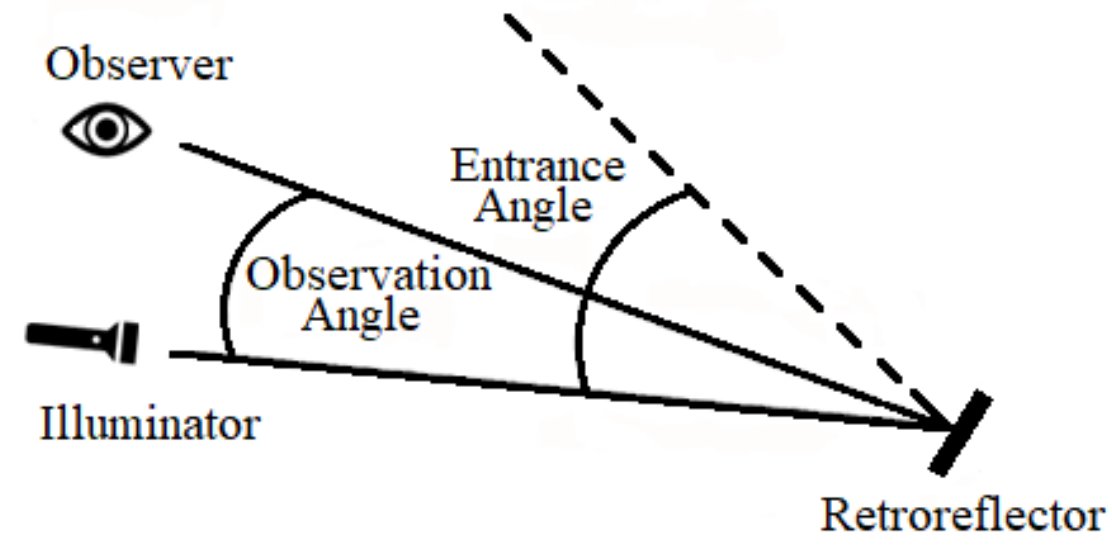

Figure 1. Observation angle and entrance angle as it applies to retroreflectors. Adapted from Federal Highway Administration (2001). Guidelines and Recommendations to Accommodate Older Drivers and Pedestrians (FHWA Publication No. 01-051). U.S. Department of Transportation. 
Observation angle is best described as the angle that separates the light source from the observer. Vehicle headlamps are strategically located just below a driver's line of sight, which allows projected light from the headlamps to come in contact with the retroreflective surface and be returned to the vehicle/driver (Uding, 1993). Rather than being spread out over a diffuse range of angles, retroreflected light returns a concentrated cone of light that maintains a high luminance when reaching the driver's eyes. The farther the driver's eyes are above their headlamps, the larger the observation angle and, consequentially, the dimmer the retroreflective material will appear to be (3M Occupational Health, 2005).

In comparison, the entrance angle is "the angle between a light source striking a surface at some point and a line perpendicular to the surface at the same point" $(3 \mathrm{M}$ Occupational Health, 2005). In the context of night driving, this means that the angle of entrance is an angle between the direction of illumination (headlamp) and an imagined perpendicular line from the location on the sign that the light came in contact with. So, for example, a road sign that is perfectly "aimed" at an approaching vehicle will have a small entrance angle but if it were inappropriately installed at an oblique angle the entrance angle would be larger. Depending on the application, retroreflective materials are designed to be robust to degradation due to wide entrance angles. Arecchi, Messadi, and Koshel (2007) explain that high quality retroreflective materials can be effective with an entrance angle up to 45 degrees or more. The reason retroreflection has been designed this way is because surfaces on a pedestrian may be located at any angle relative to an approaching motorist. Including the curvatures retroreflective clothing takes on when 
wrapped around a pedestrian's limbs, the entrance angle becomes even wider (3M Occupational Health, 2005). Specular, mirror-like surfaces are not designed with this use in mind. Specular surfaces can reflect light can back to the viewer, but only when the entrance angle is zero degrees. When not viewed under the optimal entrance angle, there is a sharp decline in illumination reflected back to the viewer surrounding this optimal entrance angle.

While the different types of reflection and the angles that are relevant to retroreflection are of critical importance to the visibility and conspicuity of potential roadway hazards at night, it is not clear that typical road users have an understanding of retroreflectivity that is deep enough to ensure that retroreflective materials will be sought out, purchased, and worn at night. Investigations of what typical road users understand about retroreflective surfaces have focused on how much brighter retroreflective surfaces appear, in comparison to diffuse reflective surfaces. The goal of the current study is to explore road users' appreciation of the other strength of retroreflective surfaces: their robustness to variations in entrance angles. We expect that some participants will exhibit a misconception that there is no functional difference between specular and retroreflective surfaces. Specular reflective surfaces only exhibit retroreflection at a narrow angle surrounding the zero degree entrance angle. This makes for an ideal comparison surface to demonstrate how robust retroreflective surfaces are to variations in entrance angle.

Since the 1970s, studies geared toward quantifying retroreflective judgments of brightness have utilized magnitude estimation (Marks, 1974; Marsh, 1998; Gravings, 
Tyrrell, \& Balk, 2009). Magnitude estimation is a psychophysical method in which participants subjectively rate the magnitude of a particular stimulus that they have experienced (Wolfe et al., 2018). By asking participants to assign a number to represent the intensity of a perceived stimulus - such as how bright an object appears - participants can compare the magnitude of other stimuli on an individualized scale. Szubski, Fekety, Edewaard, \& Tyrrell (2017) applied a magnitude estimation technique to investigate what typical road users understand about retroreflective and fluorescent materials. Perceived brightness was assessed before and during a demonstration that included illumination from a light source situated near the observer's eyes. During the pre-demonstration portion of this study, observers viewed samples of fluorescent, retroreflective, and diffuse reflective material when they were illuminated by room lights. The observers were asked to predict what the brightness of the samples would be when they were illuminated by a light source near their eyes. In the pre-demonstration (naïve) phase, observers vastly overestimated how bright fluorescent materials would appear and underestimated how bright retroreflective material would appear. A few of the colored retroreflective samples (such as black) were incorrectly understood to be of comparable brightness as their colormatched, diffuse material counterparts.

The results of Szubski, Fekety, Edewaard, \& Tyrrell's study reveal that the average participant does not understand or appreciate differences between retroreflective and diffuse reflectors in nighttime settings. However, similar to previous educational intervention findings, participants' understanding of comparative brightness for diffuse reflective and retroreflective material significantly improved once they experienced a 
visual demonstration. The current study aimed to identify further misconceptions of retroreflection. More specifically, we explored road users' appreciation for retroreflective surface's robustness to variations in entrance angle. By measuring participants' brightness judgments of different types of reflective materials at multiple entrance angles, this study sought to identify road users' misconceptions of retroreflection that might be responsible for the under-use of retroreflective markings by vulnerable road users. With this knowledge, we may now be better positioned to create more effective educational interventions.

\section{METHOD}

\section{Participants}

An a priori power analysis indicated that a sample size of 36 participants was necessary to reach a desired power of .80 or greater (medium effect size of $d=.50$ and an alpha of .05 was used). One hundred and forty undergraduate students initially participated in the study, however, only 47 of these participants were included in analyses. The ages of the 47 participants ranged from 18 to 21 years old, with 18.9 as the mean age. All 47 participants reported ownership of a valid driver's license and no visual pathologies. The average length of driver's license ownership was 3.1 years. Use of corrective lenses were reported by 13 participants (27.1\%). All participants from the original sample were rewarded with course credit toward their chosen Psychology class. Approval of the following procedures were received from the Clemson University Institutional Review Board prior to data collection. 


\section{Design}

This online study featured a within-subjects design with three independent variables. Manipulations were made to the type of surface reflectivity (specular, diffuse, or retroreflective), entrance angle level $\left(2^{\circ}, 23^{\circ}\right.$, or $\left.45^{\circ}\right)$, and presentation phases (predemo or demo). All observers experienced the pre-demonstration phase prior to the demonstration phase. Within each phase, the order in which participants experienced the nine combinations of surface reflectivity and entrance angle were randomized for each participant. The dependent variable in this experiment was the numerical estimate (magnitude estimation) of the brightness participants reported for the material sample in each of the 18 conditions.

\section{Materials}

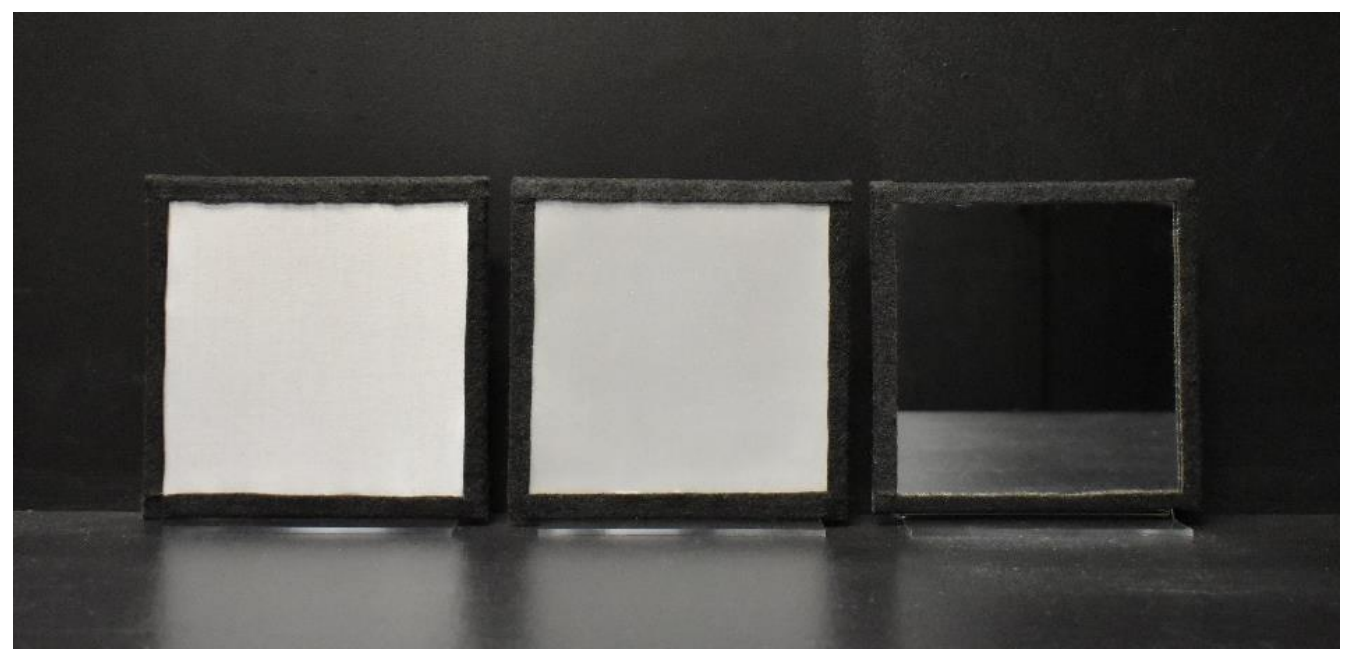

Figure 2. Experimental material samples pictured from left to right: diffuse reflective cloth, retroreflective beaded material, and specular reflective mirror.

Data collection occurred online. Qualtrics Survey Software displayed videos and documented participant responses. The survey contained video recordings of the three 
material samples $(10.2 \mathrm{~cm} \times 10.2 \mathrm{~cm})$, individually mounted to a firm, plastic backing (Figure 2). One sample was a glass mirror (specular reflector), one was cloth material (diffuse reflector), and the last was beaded retroreflective material (Safe Reflections 1099 Silver Reflective Material). All three samples had black felt borders ( $1 \mathrm{~cm}$ in width) and were silver in appearance. The diffuse and retroreflective materials were perceptually color matched.

Video stimuli were recorded using a Nikon D5600 Digital SLR Camera. An 18$55 \mathrm{~mm}$ camera lens was used and the resolution of the camera was 24.2 megapixels. All videos were recorded with a focal length of $18-24 \mathrm{~mm}$ and were displayed within the survey at a resolution of 1080p (1920 x 1080). All audio recordings and video stimuli were directly embedded within Qualtrics. Instructional videos were inserted using a thirdparty video service (Vimeo). This was done to circumvent Qualtrics' 16 MB size limit for embedded videos. The videos uploaded to Vimeo were kept private and all ads and banners were disabled at the time of data collection. Participants did not leave Qualtrics to view the videos; the videos and audio were integrated within the survey.

Eighteen videos were recorded, each exactly 10 seconds in length. Recordings of material samples featured each sample centered and displayed in an upright, 90-degree position relative to the recording camera. Samples were displayed at a distance of 2 feet $(0.61$ meters $)$ from the lens. Entrance angles $\left(2^{\circ}, 23^{\circ}\right.$, or $\left.45^{\circ}\right)$ were achieved by rotating the samples such that the right edge was brought closer to the camera than the left edge. Measurements for the correct angle and positioning of the sample on the table were subtly demarcated with black masking tape prior to the recordings. 
Before the demonstration, samples were exposed to normal room illumination from above and an approximate 271 lux illuminance was recorded. During the demonstration, illuminance was approximately 83.2 lux. When measured from the camera's position, a luminance of $9.54 \mathrm{~cd} / \mathrm{m}^{2}$ (preliminary measurement; verification pending) was found for the 2-degree diffuse sample. During the demonstration phase, an LED illuminator (ESDDI) above the camera served as the only source of illumination in the videos (Figure 3). Matte black paper was affixed to the wall and floor within the visible background of the recordings. Two free-standing, matte black backdrops at a height of 59 inches (150 centimeters) were placed behind the recording camera to eliminate visual distraction within the specular reflector conditions. Positioning of the wall, floor, and free-standing backdrops remained consistent across all conditions.

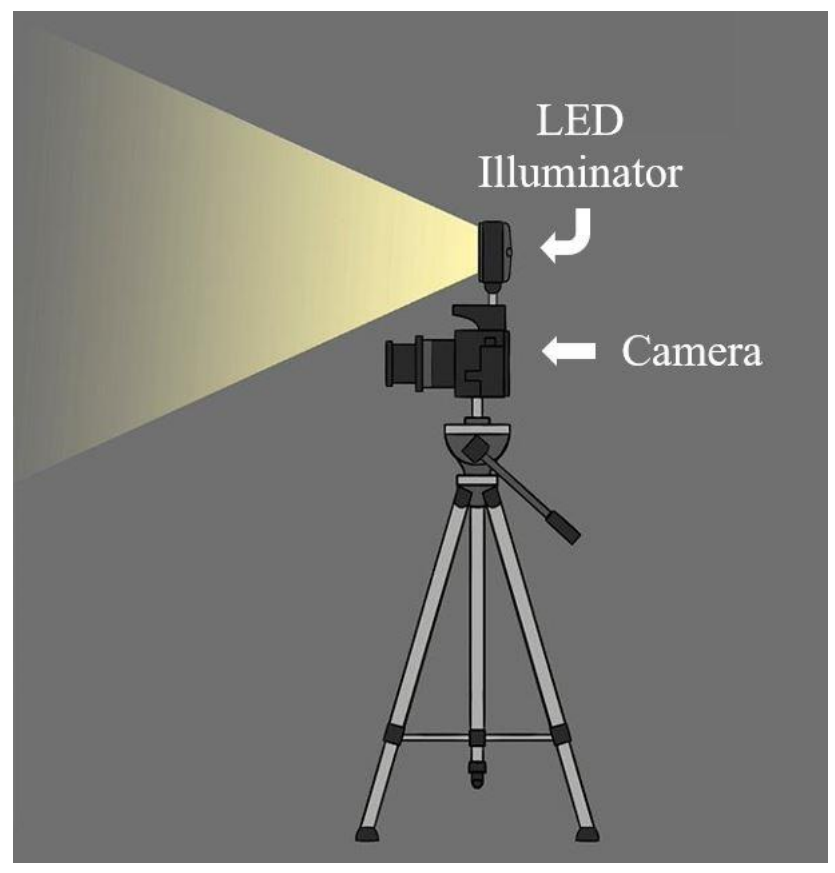

Figure 3. Location of LED illuminator during the demonstration phase, in relation to the recording camera. 


\section{Procedure}

The survey began with a set of demographic questions (see Appendix A) and audio instructions for participants to adjust the volume on their device (Appendix B). A numerical password was delivered within this 30 -second audio recording, ensuring that only those able to listen to the proceeding videos would continue with the study. A threeminute video recording then briefly described the goal of the current research study and what the study task would be (see Appendix C for the script and Appendix D for video screenshots). Closed captioning was included, and a mixture of video recordings, animation, and voice-over was used to clearly introduce the study.

To begin the pre-demonstration phase of the experiment, directions for the magnitude estimation task were delivered in text and audio form (Modified from Szubski et al., 2017; Marks, 1974):

"For each video in Part 1, your job will be to come up with a number that represents the brightness that you predict the square will have when you see it in Part 2, when the square is illuminated by the lamp. Then, for each successive video, assign other numbers in proportion to the first square's brightness. If you think the second square will be $3 \mathrm{x}$ brighter than the first square, please assign a number that is $3 \mathrm{x}$ larger. If you think the second square will be half as bright as the first square, assign a number that is half as large. Any type of positive number, whole number, decimal, or fraction may be used. Do not use zero or negative numbers. Each square will be presented for a maximum of 10 seconds. The video may be replayed as many times as you would 
like, but it is best to respond quickly and come up with a number based on your first impression."

To confirm task comprehension, participants were asked if they understand the instructions. Reworded instructions were provided for those who indicate they do not understand. Those who reported understanding the instructions then answered seven comprehension questions: two questions related to their understanding of the study set-up and five questions related to the magnitude estimation task. These questions were a combination of multiple choice and free response (see Appendix E). Although participants were not told if they answered the comprehension questions correctly, the previous set of questions were followed with a final question designed to solidify their understanding ("Do you understand that any number greater than zero is valid?").

In the pre-demonstration phase of the video recordings, the overhead room lights remained on and the illuminator was off. After viewing a 10-second video clip of one sample under normal room illumination, participants entered their "pre-demo" numeric prediction of how bright the sample would have appeared if the room lights had been off and a camera illuminator aimed at the sample had been on (Appendix F). This process was repeated for all three sample types (specular, diffuse, and retroreflective) at each of the three entrance angles. No description of the type of material was shared with the participant. Instead, the three sample types were consistently labelled as "Material A," "Material B," or "Material C" to aid participants in correctly recognizing the same sample type under various conditions. Entrance angle was also disclosed via overlayed 
text within each of the 18 video conditions, both in words and as an exact measurement (Figure 4).

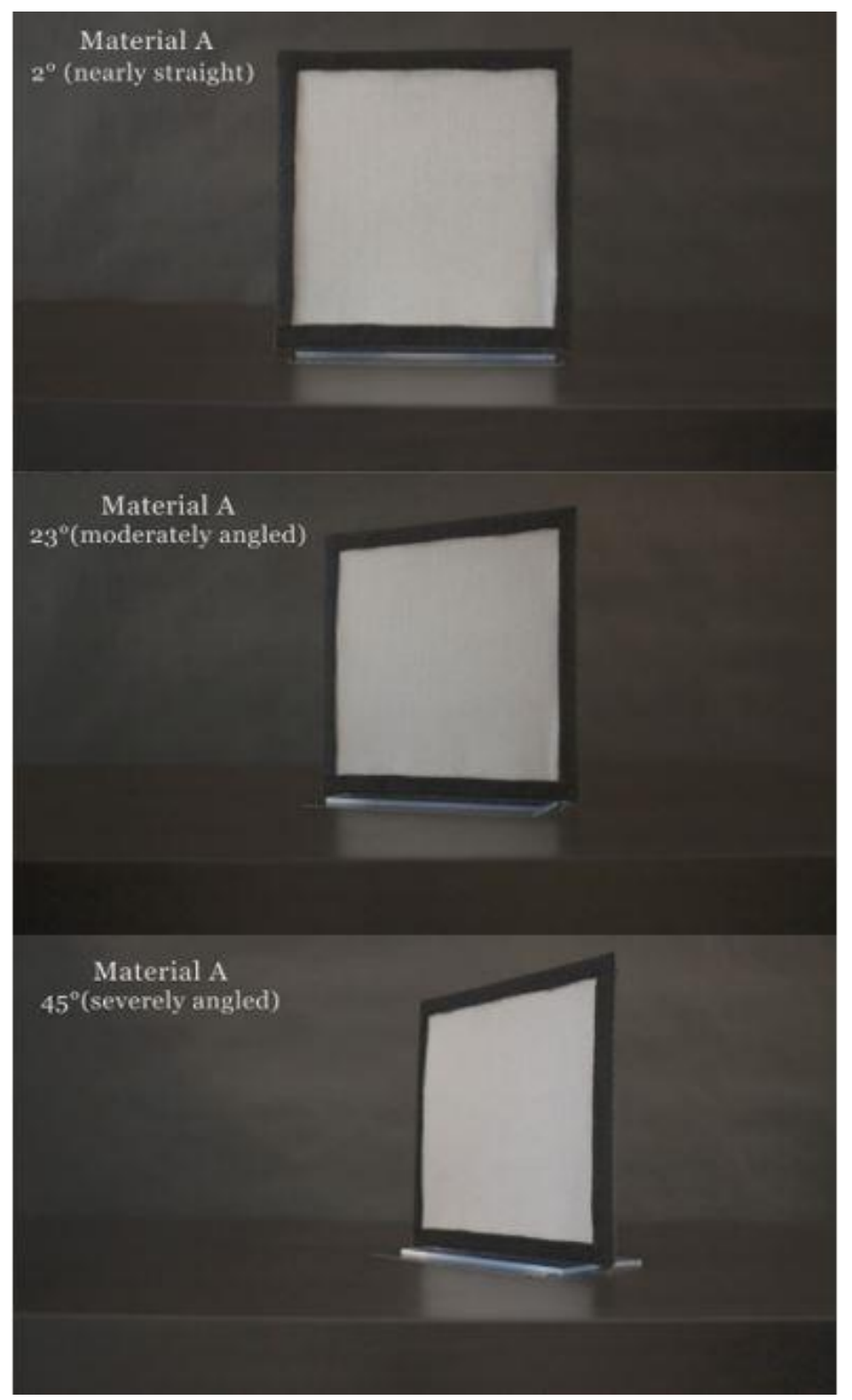

Figure 4. The diffuse material sample viewed at each of the three entrance angles, as displayed to participants during the pre-demonstration phase of the experiment.

After pre-demonstration magnitude estimates were reported for the nine sample configurations, participants proceeded to viewing the demonstration videos. The 
directions for the magnitude estimation task were repeated, with minor amendments to reflect the task differences in the Part 2:

"For each video in Part 2, your job is to come up with a number that represents the brightness that the square has right now. Then, for each successive video, assign other numbers in proportion to the first square's brightness. If you think the second square is $3 x$ brighter than the first square, please assign a number that is $3 x$ larger. If you think the second square is half as bright as the first square, assign a number that is half as large. Any type of positive number, whole number, decimal, or fraction may be used. Do not use zero or negative numbers. Each square will be presented for a maximum of 10 seconds. The video may be replayed as many times as you would like, but it is best to respond quickly and come up with a number based on your first impression."

During the recording of the demonstration videos, the overhead room lights were turned off and the camera illuminator was turned on and aimed straight ahead. An observation angle of approximately eight degrees separated the illuminator from the camera lens. Three samples were displayed one-by-one in separate 10-second videos at each of the three entrance angles. Participants then provided brightness judgments for each of the trials within the demo videos (Appendix F).

Once the experimental portion of the survey was complete, participants answered two questions related to their understanding of the study and four questions related to their knowledge of pedestrian clothing conspicuity and reflective properties (Appendix 
G). Two of the six questions were free response and the other four were multiple choice or yes-no questions.

\section{RESULTS}

Forty-seven participants were included in analyses, however, a total of 140 undergraduate students participated in the experiment. Of the 93 excluded participants, a majority (67 participants; $72 \%$ of the 93 exclusions) were disqualified for failing to meet the criteria for passing the comprehension questions. A 100\% accuracy score was required in order to be included in analyses. The time that participants took to complete the 25-minute survey was also considered. Research on magnitude estimation consistency indicates that estimates of luminance may become less reliable when given over larger spans of time, due to forgetting the constraints from the original estimations (Teghtsoonian \& Teghtsoonian, 1983). Accordingly, Qualtrics Meta Info was used to collect information on participants' survey completion time and a one-hour time limit was set. Eight of the 93 excluded participants (8.6\%) were removed from analyses for taking more than an hour to complete the study.

Embedded data were also used to preclude participation from mobile phone users. In order to promote more consistent visual angles, the survey could only be accessed by participants who were using desktop or laptop computers. Although 14 participants attempted to start the survey using a mobile device, all participants later accessed the survey using an appropriate device type and were not excluded for this reason. Other excluding factors included: indication of not fully understanding their task (7.5\% or 7 participants; Appendix G), not completing the survey entirely (4.3\%, 4 participants), self- 
reported visual pathology (3.2\% or 3 participants; Appendix A), not owning a valid driver's license (2.2\% or 2 participants; Appendix A) and not passing the computer audio check (2.2\% or 2 participants; Appendix B).

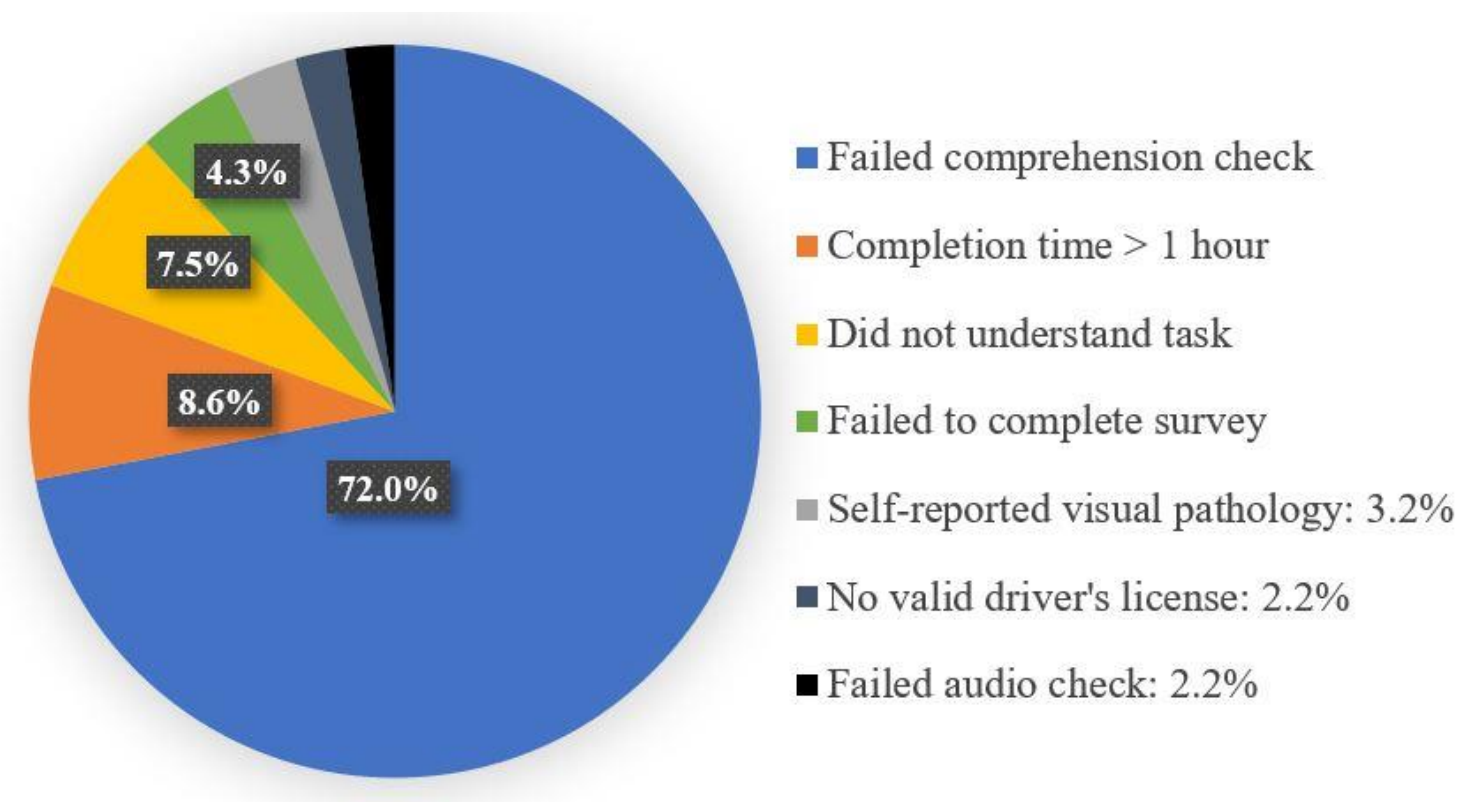

Figure 5. Ninety-three participants excluded from analyses, represented by the percent excluded for each reason.

\section{Magnitude Estimation Task}

Two terms are used throughout reporting the results of the magnitude estimation task: brightness predictions and brightness judgments. "Brightness predictions" refer to the magnitude estimation values provided during the pre-demonstration phase, where participants predicted how bright the sample would look when the room lights were off and the illuminator was on. "Brightness judgments" are the magnitude estimates that were given during the visual demonstration. These values indicated the intensity of brightness participants perceived while viewing the sample under nighttime conditions. 
Each participant contributed 18 magnitude estimates during the experimental task. These estimates were subjective ratings used to measure the predicted brightness (in the pre-demo trials) or the judged brightness (during the demo trials) of the three materials. A standardized scale was not used across participants, meaning that transformations to the raw data needed to be performed in order to analyze and compare their magnitude estimates. Geometric averaging is recommended for magnitude estimation data because it preserves the ratios between an individual participants' estimations of brightness, while remaining resilient to more extreme values produced by participants who chose a rating scale that was substantially less than or greater than the rest of the sample (Marks, 1974). This geometric averaging procedure began with utilizing $\log _{10}$ transformations to normalize participant data, similar to the arithmetic averaging method. Once a uniform scale had been created, however, each participants' mean logarithmic score was subtracted from the total mean score produced by all participants, producing their individual offset value (McGee, 2003). These offset values were then added to the individual $\log$ scores and the antilogs of the individual scores were computed, completing the geometric averaging process. The scale produced by this process resulted in the lowest averaged magnitude estimation value being 1.463 and the highest value being 9.098 (see Table 1 for more details). 
Table 1. Geometric means of the magnitude estimates for each condition.

\begin{tabular}{|c|c|c|c|}
\hline Presentation Phase & Entrance Angle & Reflectivity Type & Brightness Estimate \\
\hline \multirow{9}{*}{ Pre-demonstration } & \multirow{3}{*}{$2^{\circ}$} & Diffuse & 6.547 \\
\hline & & Retro & 6.062 \\
\hline & & Specular & 9.098 \\
\hline & \multirow{3}{*}{$23^{\circ}$} & Diffuse & 6.134 \\
\hline & & Retro & 6.376 \\
\hline & & Specular & 6.643 \\
\hline & \multirow{3}{*}{$45^{\circ}$} & Diffuse & 5.478 \\
\hline & & Retro & 5.612 \\
\hline & & Specular & 6.660 \\
\hline \multirow{9}{*}{ Demonstration } & \multirow{3}{*}{$2^{\circ}$} & Diffuse & 3.094 \\
\hline & & Retro & 6.013 \\
\hline & & Specular & 6.876 \\
\hline & \multirow{3}{*}{$23^{\circ}$} & Diffuse & 3.271 \\
\hline & & Retro & 6.689 \\
\hline & & Specular & 1.466 \\
\hline & \multirow{3}{*}{$45^{\circ}$} & Diffuse & 3.627 \\
\hline & & Retro & 7.189 \\
\hline & & Specular & 1.463 \\
\hline
\end{tabular}

Once transformations were complete, magnitude estimates were initially analyzed using a 3 (type of surface reflectivity) by 3 (level of entrance angle) by 2 (presentation phase) repeated-measures ANOVA. Mauchly's Test of Sphericity indicated that the assumption of sphericity had been violated, $\chi^{2}(9)=111.864, p<.001$, and a GreenhouseGeisser correction was applied to the degrees of freedom. After a significant three-way interaction was established, two 3 (surface reflectivity) by 3 (entrance angle) ANOVAs were used to separately assess the interaction effects within the pre-demonstration and demonstration phases. Greenhouse-Geisser and Huynh-Feldt corrections were used when necessary. Multiple paired-sample t tests were then conducted to determine what effect the visual demonstration had on estimates of brightness. Wilcoxon Signed Rank Tests were used for all subsequent $t$ tests, due to failed assumption of normality. Pre- 
established hypotheses determined which t tests were conducted in order to reduce the possibility of a type 1 error. All partial eta squared values were interpreted using Cohen's (1988, p. 283) effect size parameters (small $=.0099$, medium $=.0588$, and large $=.1379)$.

\section{Brightness Predictions}

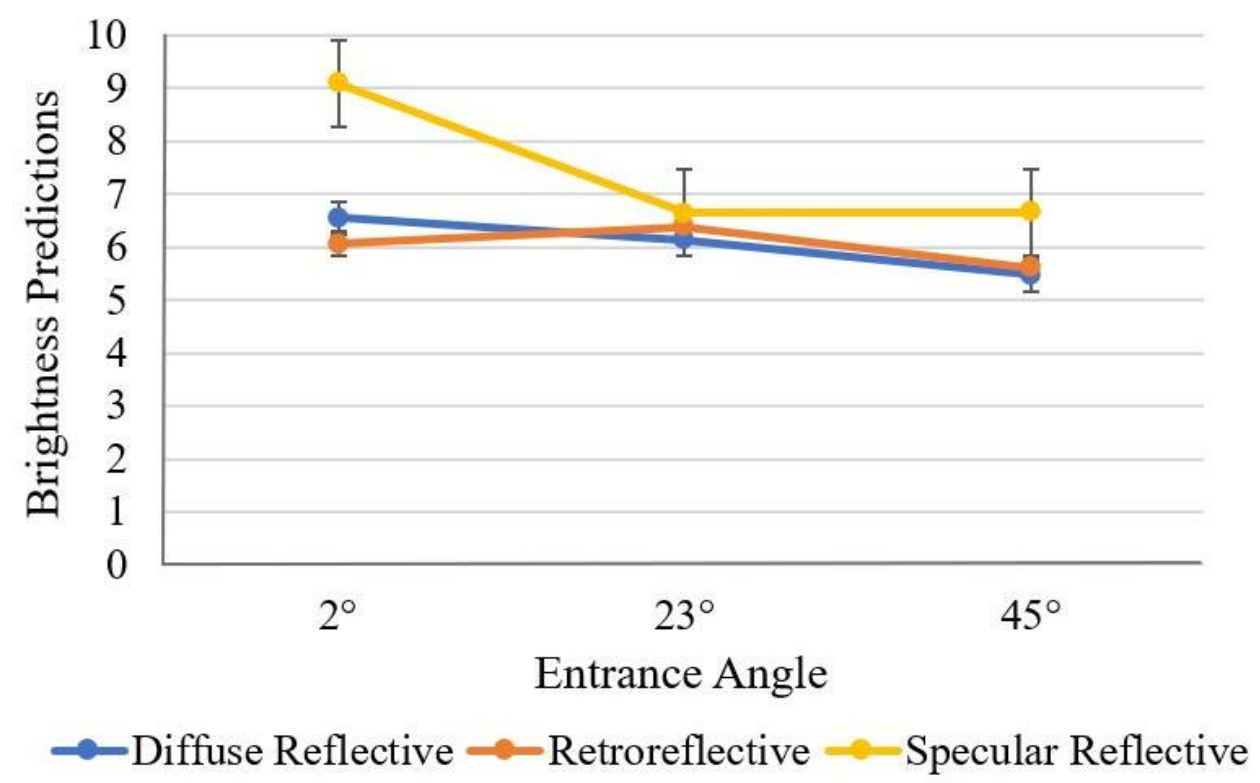

Figure 6 . Geometric mean ( \pm 1 standard error of the mean) brightness predictions during the pre-demonstration phase, as a function of entrance angle and reflectivity type.

A statistically significant three-way interaction was found, $F(1.747,46)=$ $11.495, p<.001, \eta_{p}^{2}=.20$ (large effect size). A follow-up two-way, repeated measures ANOVA revealed a main effect of entrance angle level on the pre-demonstration brightness predictions, $F(1.681,46)=5.985, p=.006, \eta_{p}^{2}=.115$ (medium effect size). Reflectivity type was also found to have a significant main effect on the pre-demo estimates, $F(1.238,46)=7.751, p=.004, \eta_{p}^{2}=.144$ (large effect size). More importantly, there was a significant interaction between entrance angle and reflectivity 
type during the pre-demonstration, $F(1.982,46)=4.975, p=.009, \eta_{p}^{2}=.098($ medium effect size; Figure 6). This indicates that participants' brightness predictions significantly varied depending on what combination of entrance angle and sample reflectivity type they were rating.

Further analysis indicates that the effect of entrance angle on pre-demonstration brightness predictions were found to be significant for both the specular sample, $F(2,46)$ $=6.740, p=.002, \eta_{p}^{2}=.22$ (large effect size), and the diffuse sample, $F(2,46)=4.572, p$ $=.013, \eta_{p}^{2}=.166$ (large effect size). Entrance angle level did not significantly impact the brightness predictions for the retroreflective sample, $F(2,46)=1.754, p=.179, \eta_{p}^{2}=$ .071 (medium effect size). Participants predicted the retroreflector during the demo would be of similar brightness across all three entrance angles, whereas the specular and diffuse samples would exhibit changes in brightness as the level of entrance angle was manipulated. A paired-samples $t$ test revealed that participants also expected the retroreflector and diffuse reflector to have similar brightness during the visual demonstration, $t(46)=0.086, p=1.00,95 \%$ CI $[-0.988,1.061]$, Cohen's $d=0.013$ (small effect size; see Figure 6).

Reflectivity type significantly impacted brightness predictions at two of the three entrance angles: 2 degrees, $F(2,46)=9.213, p<.001, \eta_{p}^{2}=.286$ (large effect size), and 45 degrees, $F(2,46)=6.608, p=.002, \eta_{p}^{2}=.223$ (large effect size). However, there was no effect of reflectivity type in the 23-degree entrance angle level, $F(2,46)=0.491, p<$ $.613, \eta_{p}^{2}=.021$ (see Figure 6 ). This suggests that participants primarily considered the 
type of material that they were viewing to be relevant when making predictions within the 2-degree and 45-degree conditions, but not the 23-degree condition.

\section{Brightness Judgments}

While analyzing the brightness judgments that were recorded during the demonstration, a two-way ANOVA revealed a significant interaction between entrance angle and reflectivity type with a much larger effect size present than in the pre-demo, $F$ $(1.097,46)=22.871, p<.001, \eta_{p}^{2}=.332$ (large effect size; Figure 7). A significant main effect of entrance angle on demonstration brightness judgments was also present, $F$ $(1.125,46)=10.737, p=.001, \eta_{p}^{2}=.189$ (large effect size). In addition, brightness judgments during the demonstration showed a significant main effect of reflectivity type, $F(1.348,46)=40.014, p<.001, \eta_{p}^{2}=.465$ (large effect size). Comparing pre-demo brightness predictions to the judgments made during the visual demo illustrates that participants poorly anticipated the perceived brightness of material samples based on their reflectivity type (Figure 8) and entrance angle (Figure 9). 


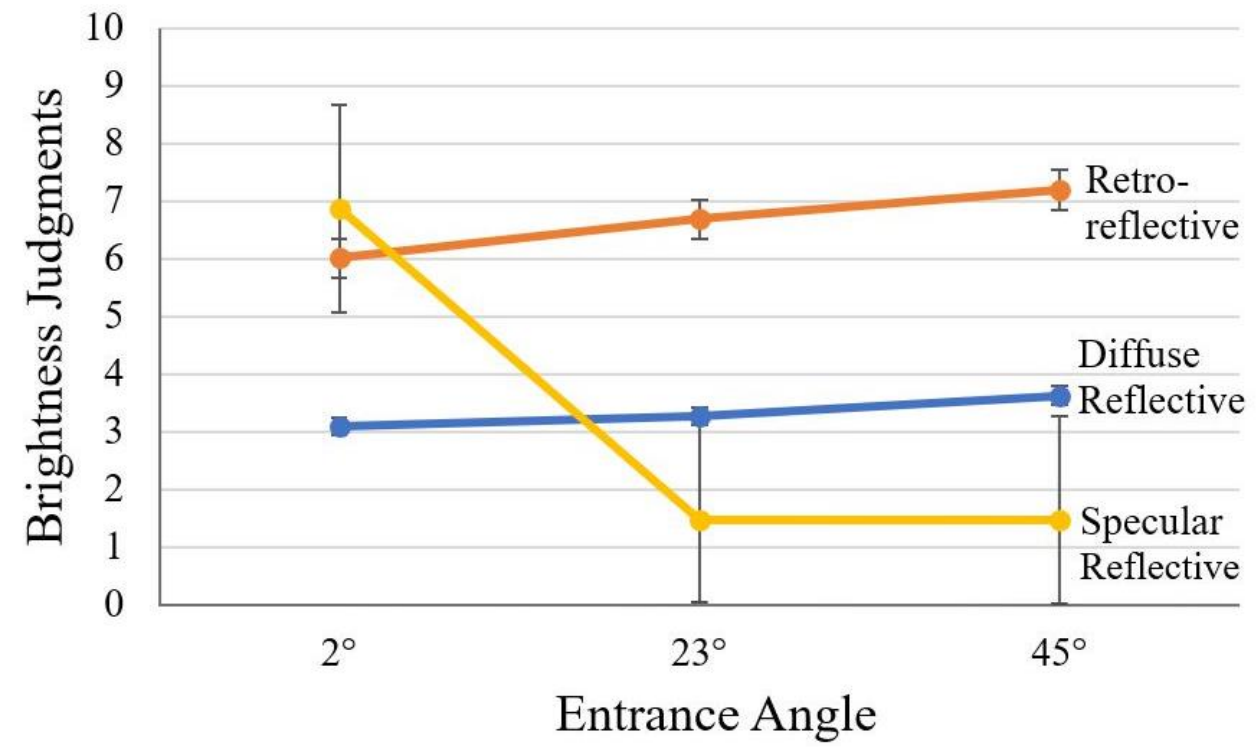

Figure 7. Geometric mean ( \pm 1 standard error of the mean) brightness judgments during the demonstration as a function of entrance angle and reflectivity type.

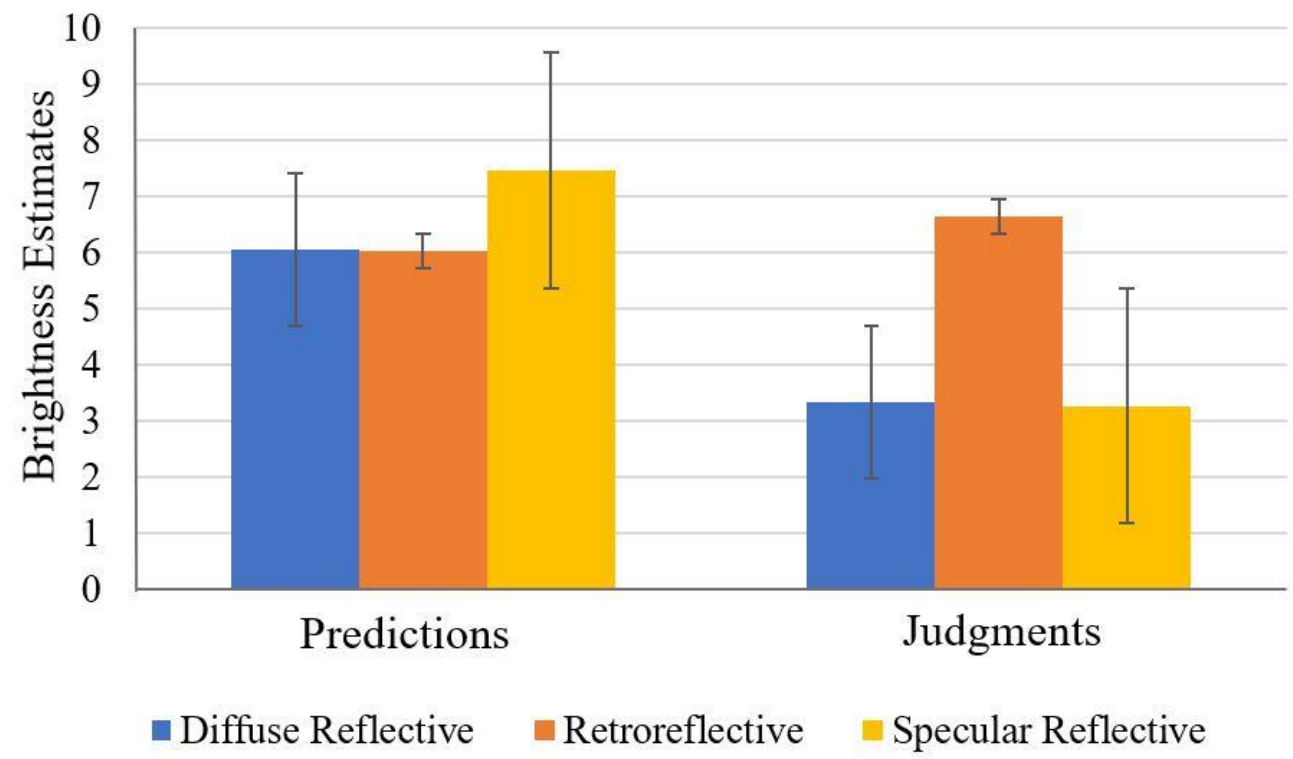

Figure 8 . The effect of reflectivity type on pre-demonstration brightness predictions and demonstration brightness judgments. These data are averaged across the three entrance angles. Error bars represent \pm 1 standard error of the mean. 


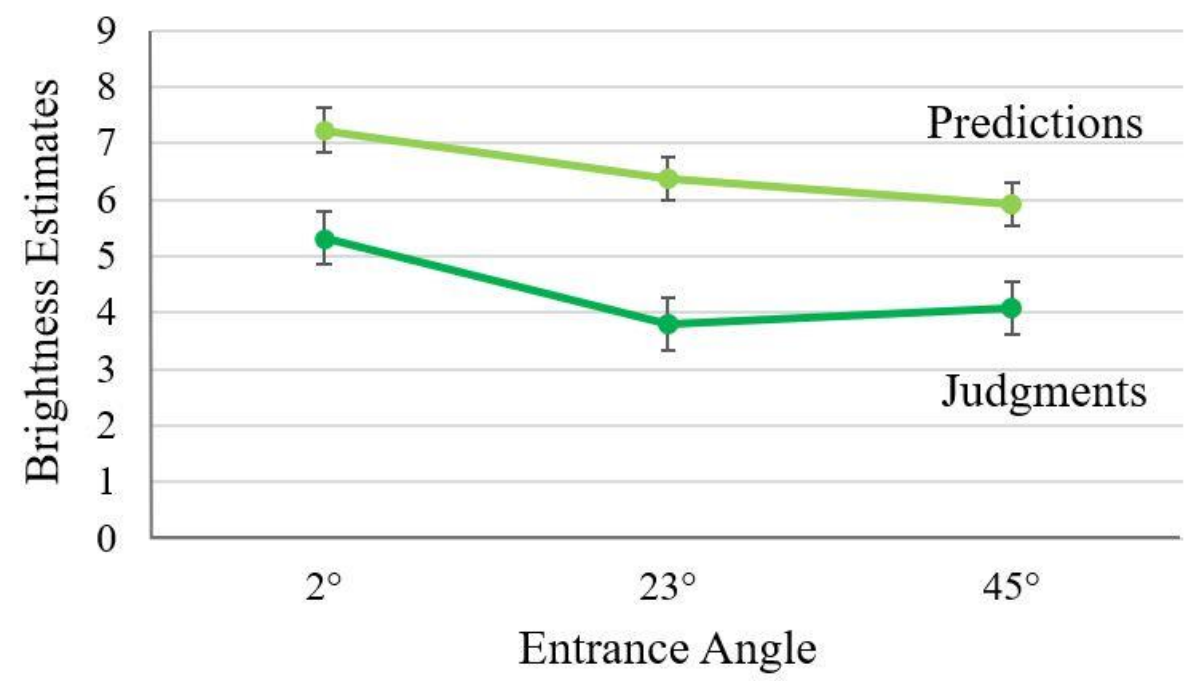

Figure 9. The effect of entrance angle on pre-demonstration brightness predictions and demonstration brightness judgments, when averaged across reflectivity type. Error bars represent \pm 1 standard error of the mean.

To determine what effect the visual demonstration had on specular estimates of brightness, multiple paired-sample $t$ tests were conducted. The specular surface was expected to spike at a nearly-straight entrance angle $\left(2^{\circ}\right)$ during the demo, because two degrees is near the optimal entrance angle at which specular reflective surfaces retroreflect incoming light $\left(0^{\circ}\right)$. A paired-samples $t$ test revealed that the specular surface did receive magnitude estimations that were significantly higher at the 2-degree entrance angle $(M=6.876, S D=9.023)$ than at 23 degrees $(M=1.466, S D=1.194), t(46)=$ $1081.00, p<.001,95 \% \mathrm{CI}[1.00, \infty], r=1.00$. Comparing the specular sample at two degrees $(M=6.6876, S D=9.023)$ versus 45 degrees $(M=1.463, S D=1.413)$ also indicated that there was a very strong effect of entrance angle on specular sample brightness evaluations during the demonstration, $t(46)=1081.00, p<.001,95 \% \mathrm{CI}$ 
$[1.00, \infty], r=1.00$. No significant difference was found for the specular sample when comparing brightness judgments at 23 degrees $(M=1.466, S D=1.194)$ and 45 degrees $(M=1.463, S D=1.413), t(46)=107.00, p=.078,95 \%$ CI $[-.236, \infty], r=.399$. All three of these comparisons yielded expected results, due to the brightness of the specular reflector plummeting at the two more extreme entrance angles (Figure 10). Once the entrance angle of the specular sample was no longer near zero degrees, the optimal angle for retroreflection, incoming light was reflected in a direction away from the camera.

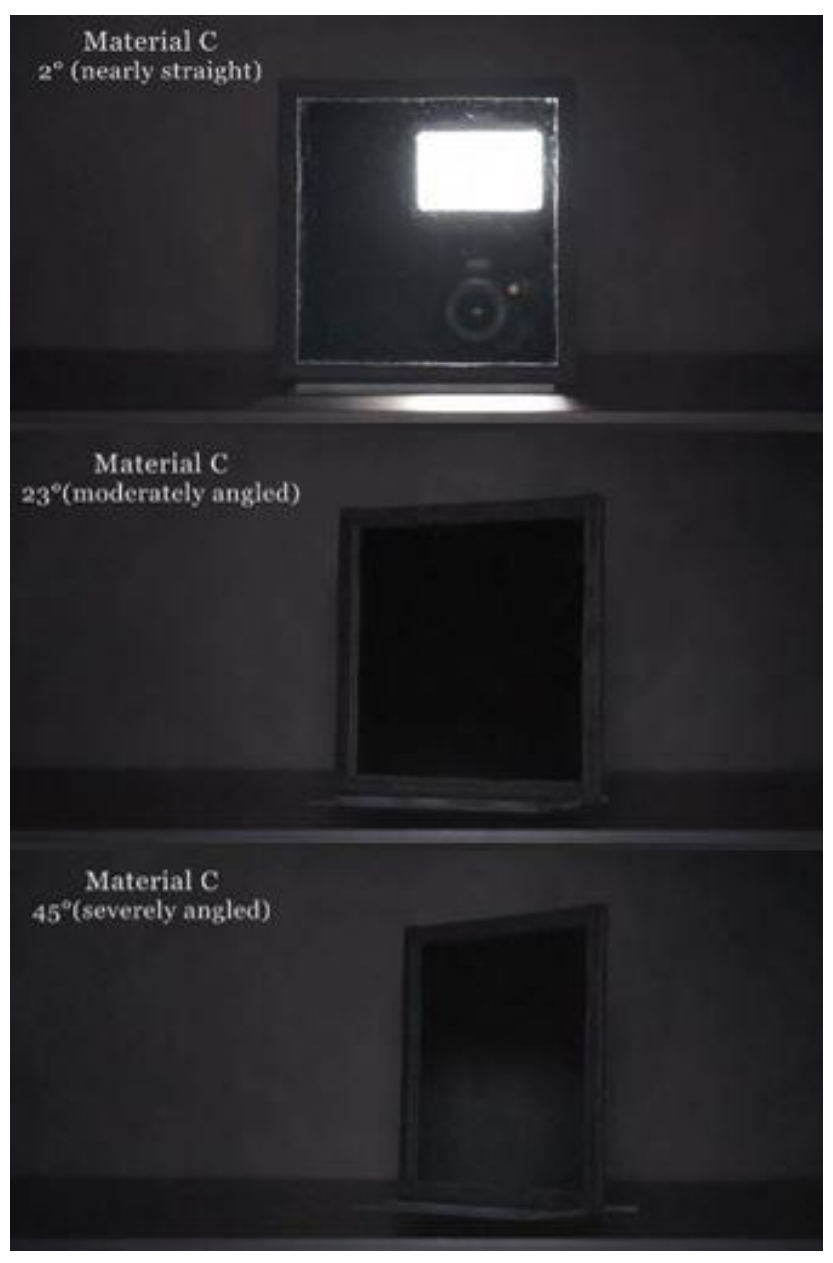

Figure 10. Specular reflective samples displayed with an entrance angle of 2 degrees, 23 degrees, and 45 degrees. 
While watching the visual demonstration with the entrance angle set to 45 degrees, participants reported retroreflective material retaining (and surpassing) its anticipated maximal brightness in the 2-degree condition. A pairwise comparison of the retroreflective sample at 45 degrees $(M=7.189, S D=6.520)$ and at 2 degrees $(M=$ 6.013, $S D=5.908)$ indicated that the retroreflective material was reported as being significantly brighter in the 45 -degree entrance angle, $t(46)=522.00, p<.001,95 \% \mathrm{CI}$ $[0.437, \infty], r=.657$. A smaller but significant effect could be seen during the demonstration for the diffuse reflective material. The diffuse sample received higher brightness judgment values when under the most extreme entrance angle $\left(45^{\circ} ; M=3.627\right.$, $S D=3.473)$ in comparison to the least extreme entrance angle $\left(2^{\circ} ; M=3.094, S D=\right.$ 3.357), $t(46)=386.00, p=.03,95 \% \mathrm{CI}[0.067, \infty], r=.376$. Brightness retention as the retroreflective material increased in entrance angle was unsurprising because of the material's resilience to extreme entrance angles. Even so, an increase in brightness judgments from the 2-degree to 45-degree conditions were not anticipated for any of the samples, mostly because the visual angle of the surface had decreased (Figure 11). 


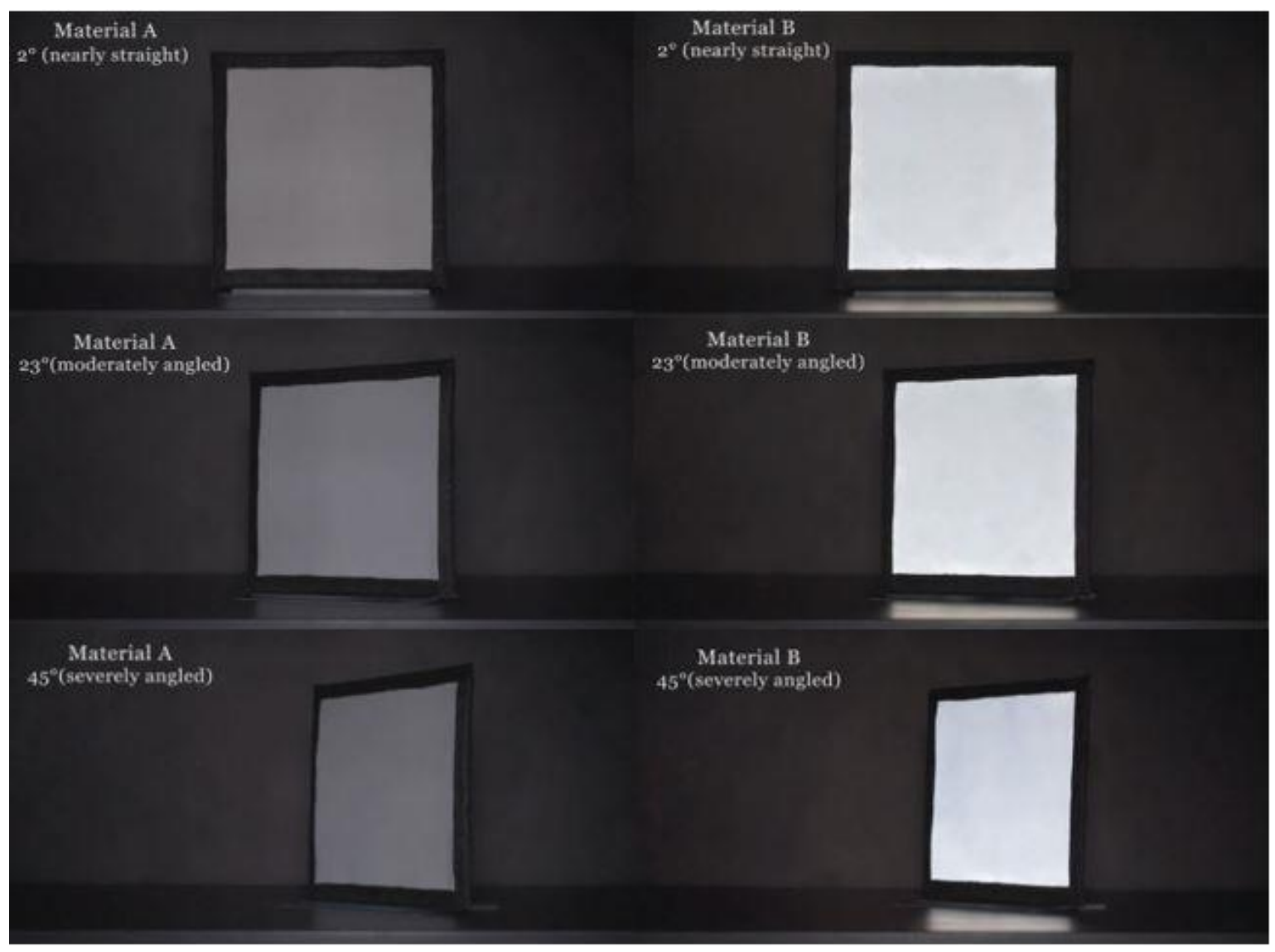

Figure 11. Comparison of diffuse (left) and retroreflective (right) samples in the 2degree, 23-degree, and 45-degree conditions during the visual demonstration.

To assess the effects of the demonstration on brightness predictions within the most extreme entrance angle $\left(45^{\circ}\right)$, multiple paired-samples $t$ tests were conducted. Viewing visual demonstrations of reflection seemed to produce an enhanced appreciation that only retroreflective surfaces remain bright despite large changes in entrance angle (see Figure 12). Estimates of brightness for the retroreflective material were significantly higher during the visual demonstration $(M=7.189, S D=6.520)$ than the predemonstration $(M=5.612, S D=6.150), t(46)=139.00, p<.001,95 \%$ CI $[-\infty,-0.552], r$ $=-.719$. The specular reflector, however, experienced a significant decrease from initial 
brightness predictions ( $M=6.660, S D=7.071)$ to later brightness judgments $(M=1.463$, $S D=1.413), t(46)=735.00, p<.001,95 \%$ CI $[0.653, \infty], r=.793$. Diffuse reflective samples also exhibited a decrease from pre-demo predictions $(M=5.478, S D=6.344)$ to demo estimates $(M=3.627, S D=3.473), t(46)=897.00, p<.001,95 \%$ CI $[0.976, \infty], r$ $=.987$.

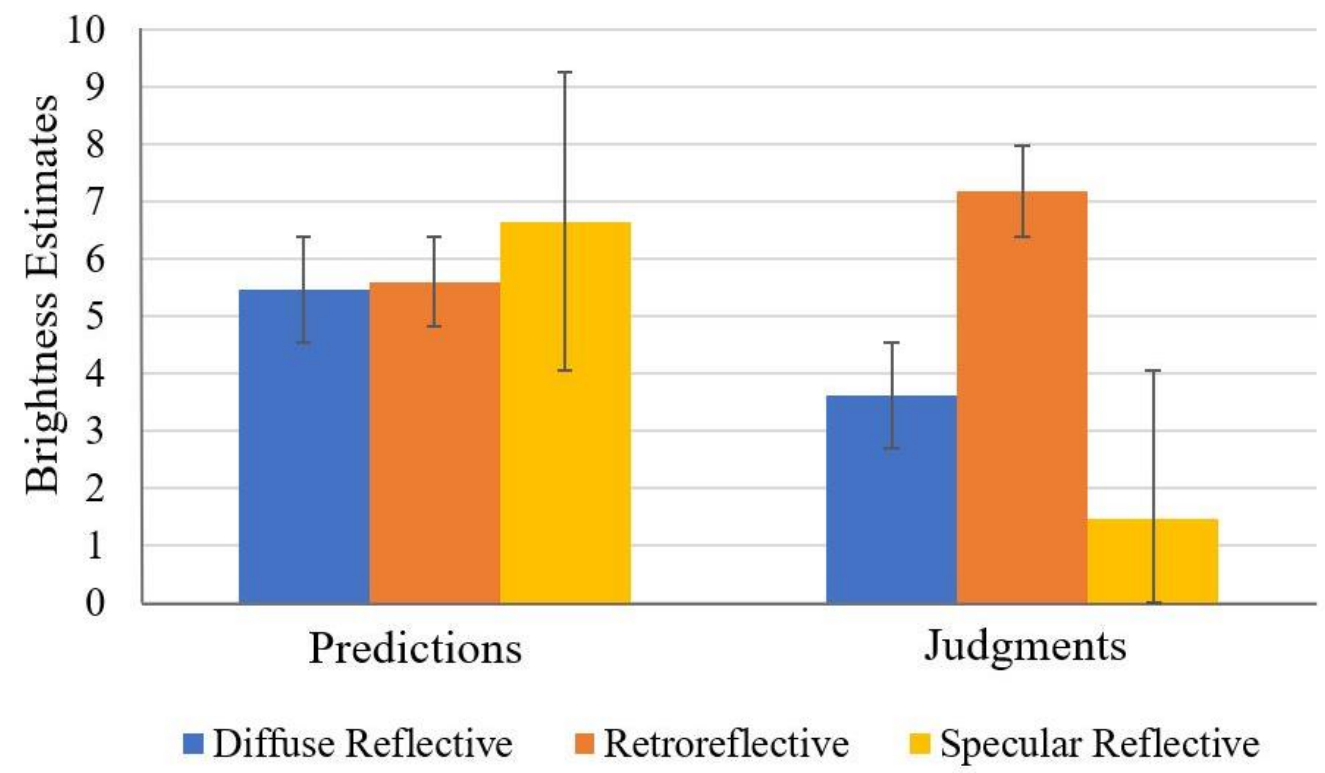

Figure 12. The effect of reflectivity type on pre-demonstration brightness predictions and demonstration brightness judgments for the 45-degree entrance angle. Error bars represent \pm 1 standard error of the mean.

Although a few of the brightness predictions participants made in the pre-demo phase varied based on the sample's entrance angle and reflectivity type, these variations tended to be small. When averaged across entrance angle and reflectivity type, the comparison of predicted brightness $(M=6.512)$ and judged brightness $(M=4.409)$ indicate that participants expected samples overall to look substantially brighter (by $32.3 \%$ ) in the nighttime conditions than they did. Inaccuracies in brightness predictions 
were even more pronounced when comparing the mean brightness of individual conditions (Figure 13). For example, the specular reflective sample at 45 degrees $(M=$ 6.659) was predicted to be approximately $18.7 \%$ brighter than the retroreflective sample $(M=5.612)$ and $21.6 \%$ brighter than the diffuse sample $(M=5.478)$ at the same entrance angle. Yet during the demonstration, the mean brightness judgment for the 45-degree, retroreflective sample $(M=7.189)$ was over 4.5 times brighter than the specular sample $(M=1.463)$. In addition, the diffuse sample $(M=3.627)$ was rated as roughly twice as bright as the specular sample.

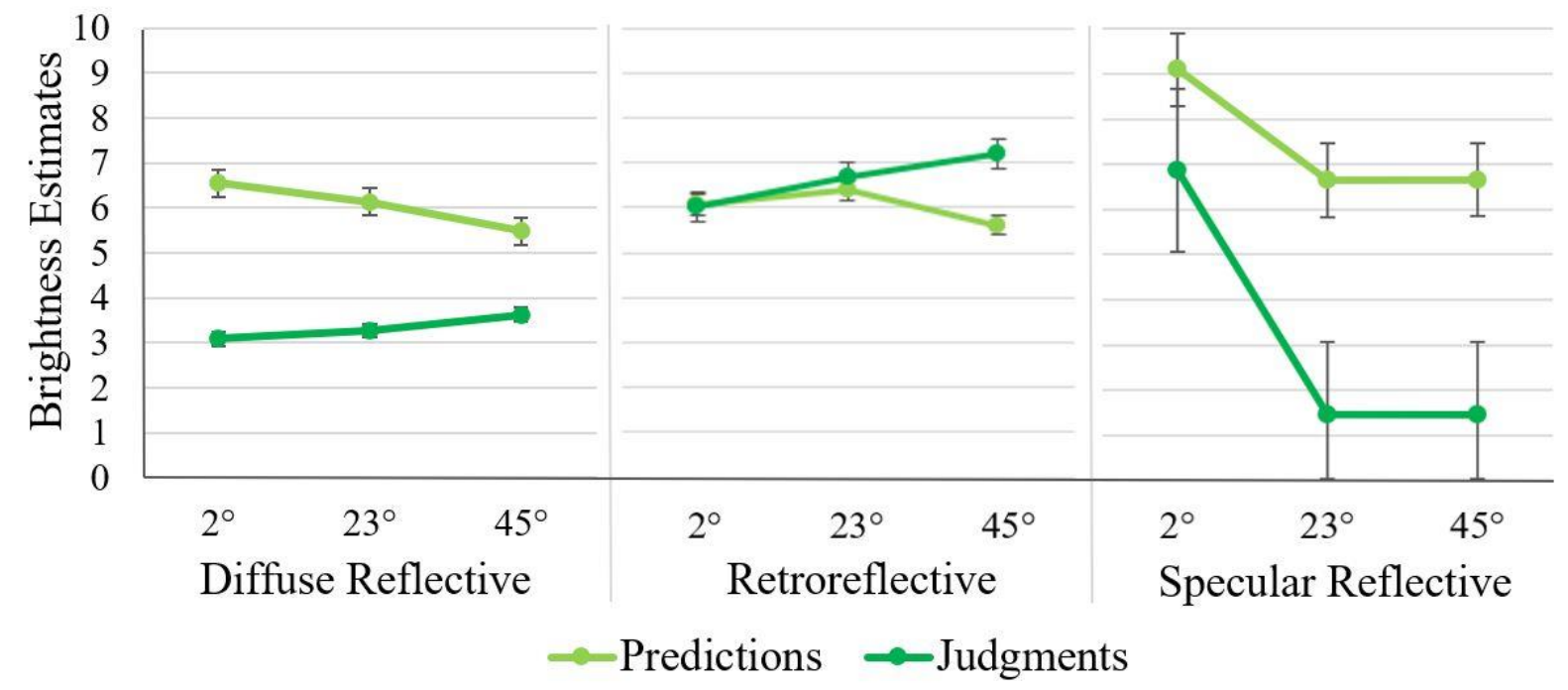

Figure 13. Geometric mean brightness for all 18 conditions as a function of presentation phase, reflectivity type, and entrance angle. Error bars represent \pm 1 standard error of the mean. 


\section{Post-experiment Survey}

At the end of the experiment, participants answered questions related to their understanding of the study and their knowledge of pedestrian clothing conspicuity and reflective properties (Appendix G). When asked about what they should wear when walking near traffic during daylight, $68 \%$ chose either fluorescent (19 participants) or a retroreflective vest (13 participants). The remaining 32\% reported that they believed white clothing to be what they should wear during daylight. For the question regarding what participants should wear while interacting with traffic at night, $74.5 \%$ (35 participants) correctly chose the most conspicuous option: the retroreflective vest. Of the remaining 12 participants, eight chose the fluorescent clothing $(25.5 \%)$, three chose the flashlight (6.4\%), and one chose the white clothing (2.1\%). When asked if they were familiar with the term "retroreflective," 26 participants $(55.3 \%)$ reported that they were familiar with the term and 21 participants $(44.7 \%)$ reported that they were not. Those who indicated being familiar with the term were then asked how the meaning of retroreflective was different from reflective.

A thematic analysis using inductive coding uncovered trends in participants' freetext responses. Detailed participant responses often included concepts that were represented within more than one theme category. Of the 26 participants who were asked to explain the difference between the terms "retroreflective" and "reflective," 14 participants (53.8\%) correctly described retroreflective materials as sending incoming light back to the source. Nine of those participants additionally expanded on this idea to say (correctly) that diffuse reflective surfaces, in comparison, send incoming light in 
different directions. Two participants (7.7\%) did not mention how the properties of retroreflection and reflection differ, however, they did mention that retroreflective materials are useful in nighttime conditions. Analyzing participant responses also revealed that four participants (15.3\%) associated the word "reflection" with having mirror-like qualities, whereas "retroreflection" did not.

The remaining participants excluded in the previous themes either answered too briefly to be categorizable ( 3 participants; $11 \%$ ) or were particularly unique ( 2 participants; 7.7\%). One participant focused on the "iridescent" quality of retroreflective material being different than "normal reflective material." Another said the difference between retroreflection and reflection is that retroreflective materials exhibit reflection "not only while the light is being shined directly on it [but also] from different angles for prolonged periods of time."

\section{DISCUSSION}

This research was motivated by the fact that too few vulnerable road users seek out, obtain, and wear retroreflective markings when they interact with traffic at night. An investigation of possible misconceptions about retroreflective surfaces could address the dissonance among what people report knowing they should wear, what they actually choose to wear, and what materials are maximally visible at night (e.g., Hagel et al., 2007, as cited in Wood et al., 2009). For this study, emphasis was placed on assessing the degree to which road users appreciate the robustness of retroreflective materials to variations in entrance angles. Taken together, the results indicate that entrance angle most significantly impacted brightness predictions for the specular reflector. While pre- 
demonstration brightness predictions for the retroreflective sample only decreased $7.42 \%$ between the nearly straight condition $\left(2^{\circ} ; M=6.062\right)$ and the severely angled condition $\left(45^{\circ} ; M=5.612\right)$, specular reflective material saw a $26.8 \%$ decrease between the 2 -degree $(M=9.098)$ and 45-degree entrance angle conditions $(M=6.660)$. Although it was not as drastic of a decline as was observed for the specular sample, diffuse material also exhibited a significant decrease of $16.33 \%$ between the nearly straight condition $\left(2^{\circ} ; M=\right.$ $6.547)$ and the severely angled condition $\left(45^{\circ} ; M=5.478\right)$. Participants seemed to have an understanding that even moderate manipulations of entrance angle would impact diffuse and specular surface brightness.

Nevertheless, participants underestimated the sensitivity of diffuse and specular reflectors to variations in entrance angle. Even within the condition with the lowest predicted brightness for the specular sample $\left(45^{\circ}\right.$ entrance angle $)$, participants incorrectly predicted that the specular sample would appear significantly brighter to them in nighttime roadway conditions than the retroreflective or diffuse samples. A possible explanation for this finding is that the appearance of specular material in average room illumination provides noticeable visual clues to signal its retroreflective capabilities, whereas this isn't as observable for retroreflective or diffuse materials. It was hypothesized that the predicted brightness for the specular sample would be influenced (and mislead) by the reflective, "shiny" appearance of specular reflectors when viewed under room illumination. This prediction arose from Szubski, Fekety, Edewaard, \& Tyrrell's (2017) finding that participants reported smaller differences between their predicted magnitude estimates and magnitude estimates during the demonstration when 
they were able to closely inspect a rigid sample of retroreflective material. If this were due to their attention being drawn to the "shimmery" appearance of beaded retroreflective materials when viewed closely, it is likely that this would also apply to how specular material was perceived during the current study's pre-demonstration phase. The "shiny" appearance in daytime conditions might have anchored participants' overall brightness predictions for specular materials at a higher magnitude, while the inability to see the reflection of the illuminator in the 23-degree and 45-degree conditions marginally reduced brightness predictions. It is also possible that these results signify a degree of geometric naivete, wherein participants are unable to predict how specular reflectors will react to manipulations of illumination source and material positioning.

A nonsignificant effect of entrance angle on predictions of the brightness of diffuse and retroreflective samples also suggests that viewing these materials under average room lighting does not offer the same visual cues that they will appear differently in nighttime conditions. The unexpected aspect of this result is that the proximity of the samples to the camera did not successfully convey to participants the special reflective properties of the retroreflective sample. It is possible that viewing the samples closely had a different effect in the online video recordings than previous research with in-person demonstrations. One explanation could be that viewing recordings of retroreflective material lacks the exploratory element of being able to manually manipulate the sample in-person. However, Szubski, Fekety, Edewaard, \& Tyrrell also found that participants' brightness predictions of rigid samples of retroreflective material presented close to the observer were similar to their brightness predictions of flexible/bendy samples that they 
were allowed to manipulate manually (2017). Investigating the cause of these conflicting results could be a starting point of future study, as it is highly relevant to the creation and success of online educational interventions (where physical manipulation of retroreflective materials is absent).

With brightness predictions averaged across entrance angle level, significant main effects were also found for surface reflectivity. The specular reflector was predicted to be the brightest (average value of 7.467), then the diffuse reflector (6.053), and the retroreflector was predicted to be the least bright (6.017). This further suggests that participants used the information available to them (appearance of sample in room illumination, material and angle labels) while making their brightness predictions. Even so, a comparison of pre-demo predictions to judgments made during the demo reveals a disparity between what was anticipated and what was later perceived. Overall, participants expected to perceive samples as being $32.3 \%$ brighter than they later reported during the demonstration (when the illuminator was the only source of light in the room). This disparity was even more pronounced when isolating specific conditions for comparison. The specular reflective sample at 45 degrees $(M=6.659)$ received brightness predictions that were $18.7 \%$ brighter than the retroreflective sample $(M=5.612)$, yet later brightness judgments indicated the retroreflective sample $(M=7.189)$ appeared over 4.5 times brighter than the specular sample $(M=1.463)$. Viewing a visual demonstration of the three surfaces significantly and substantially reduced brightness ratings for the specular and diffuse sample, but it increased ratings for the retroreflective sample. In other words, although the participants' brightness predictions were influenced by the 
material of the sample and its entrance angle, the participants failed to anticipate how the samples would appear in reduced illumination conditions that were designed to mimic nighttime roadway conditions. The data from the retroreflective material was particularly striking in that participants failed to predict that its appearance would be robust to increases in entrance angle.

\section{Implications}

Retroreflective clothing is a relatively accessible, convenient, and affordable means of enhancing pedestrian and bicyclist conspicuity at night. Research on attitudes toward high visibility clothing has shown that pedestrians report being more open to wearing retroreflection after a demonstration (Borzendowski, Sewall, Fekety, \& Tyrrell, 2014). Studies like these emphasize how simple yet powerful educational interventions are in informing pedestrians and changing their attitudes toward visibility aids. However, even when people acknowledge awareness of the more conspicuous clothing choice at night (i.e., retroreflective vest), Szubski et al. found that light colored clothing was the most frequently stated choice of garment to wear. Investigating and correcting possible misconceptions about retroreflective surfaces could address a fundamental misunderstanding of a critically useful approach to road safety at night. If so, it could help further the creation of accessible, web-based educational intervention strategies. Current methods of educating the public about nighttime road safety involve lectures from experts on visual perception at night. A more accessible and affordable solution could be to leverage video demonstrations to highlight the problem of nighttime 
pedestrian conspicuity and how valuable effectively positioned retroreflective materials can be.

Previous adaptations of in-person experimentation to online format have proved that one of the key obstacles to creating web-based educational interventions is accurately representing our nighttime perceptual experiences through video format (Balk, Brooks, Klein, \& Grygier, 2012). Ideally, our magnitude estimation results during the video demonstration should mimic those found by previous in-person data collection measuring brightness for materials used as visibility aids. A potential complication of comparing magnitude estimates from Szubski et al.'s study to the current one is that different methods of averaging were employed. Arithmetic means were used to transform the raw magnitude estimation data within the previous study, whereas the current study used geometric means. Arithmetic means are said to nearly always provide higher average estimates than geometrics means when applied to the magnitude estimation technique (Marks, 1974; Mozkowitz, 1977), and this would be particularly true for conditions that produce the most extreme values (i.e. retroreflective demonstration estimates). A comparison of the results of these two studies (see Figure 14) indicate that the averaging technique employed did not appear to significantly impact magnitude estimation data. Regardless, extra precaution was taken by applying the geometric averaging technique to Szubski et al.'s raw data. Many of the main findings from the previous study (related to magnitude estimates of silver retroreflective and silver diffuse sample brightness) were successfully replicated within the current study. 

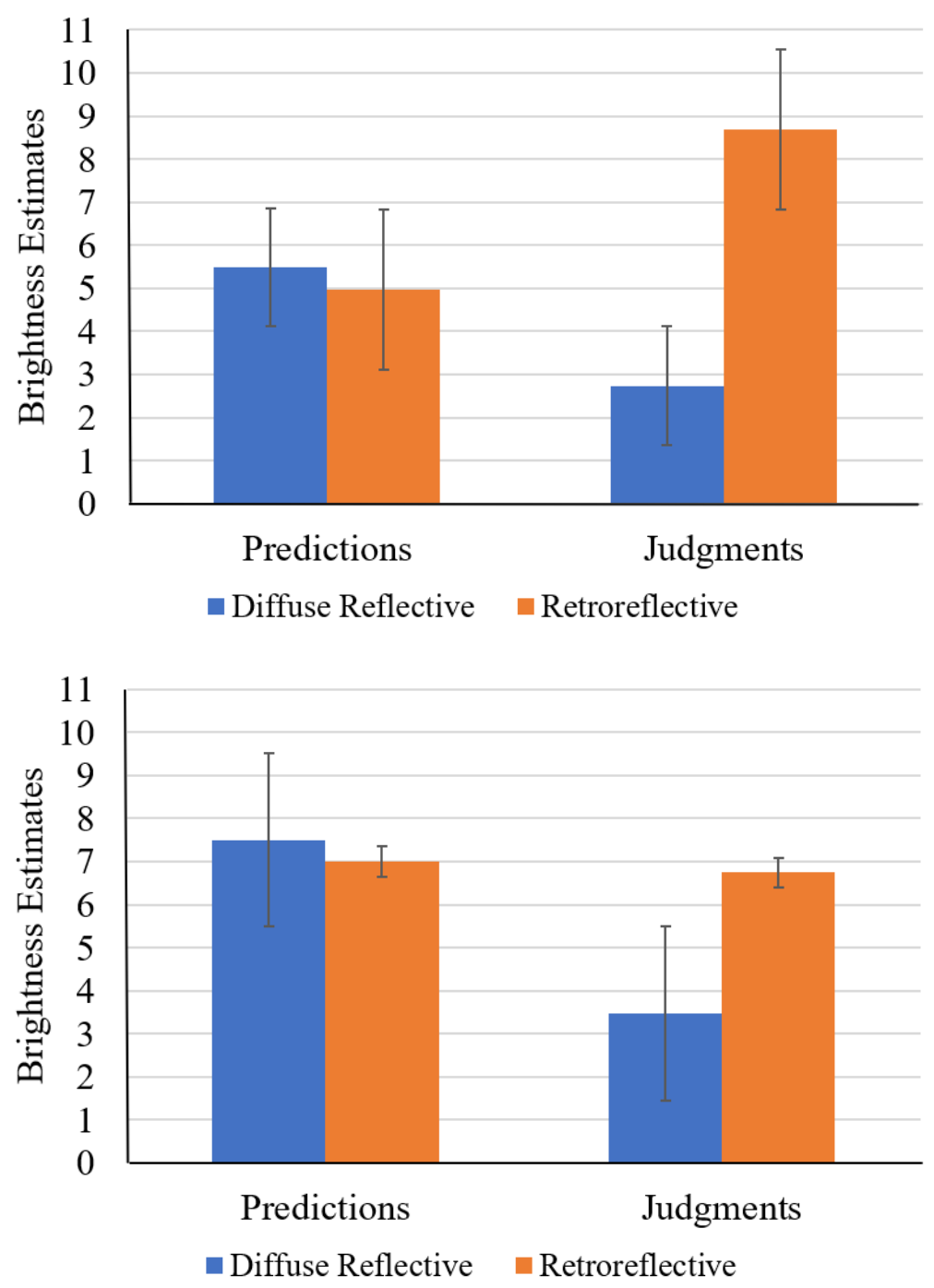

Figure 14. Top: Szubski et al.’s magnitude estimation results from an in-person demonstration of reflection with the silver samples affixed to a wall 20 feet $(6 \mathrm{~m})$ from the observers. Bottom: Magnitude estimation results from the current study's online demonstration for the 2-degree condition. Geometric means $( \pm 1$ standard error of the mean) are plotted for both studies.

The retroreflective and diffuse reflective samples within the current study were made with identical materials to those used within Szubski et al.'s silver diffuse and 
silver retroreflective conditions. To best evaluate the effectiveness of an online visual demonstration of retroreflection (current study) in lieu of an in-person demonstration (Szubski et al.'s study), the comparison of magnitude estimation results were limited to conditions using silver material. Szubski et al.'s study and the current one both found that participants viewing retroreflective materials in normal room illumination did not aid their predictions in how bright these material samples would appear in a relevant nighttime setting. Furthermore, they both discovered that the brightness of diffuse materials (such as the fabrics from which clothing is typically made) was significantly overestimated in the pre-demonstration, compared to the demonstration estimates. It was also found that online magnitude estimates were most similar to in-person magnitude estimates in the conditions where the sample was affixed to the wall (Figure 14). With online demonstrations of nighttime road conditions producing changes in estimates of brightness that are reasonably similar to those found from in-person demonstrations, there is reason for optimism regarding future success with designing video demonstrations of retroreflectivity.

\section{Limitations}

Conducting data collection online resulted in a number of limitations. One is the lack of verifiable ways to ensure participants were visually healthy. The validity of current online acuity tests is not well documented, and contrast sensitivity is even more underrepresented in this domain. As a consequence, we depended on participants' selfreport for whether they had any visual pathologies. Another demographic question aimed toward gauging visual health was regarding their ownership of a valid driver's license. 
Those who self-reported having a driver's license are assumed to have met the visual qualifications necessary to drive. Similarly, the results of the post-experiment survey about retroreflection relied on honest and unaided participation. Since there were no measures to prevent participants' use of online resources, these results should be interpreted with caution. However, the fact that each of the manipulations in the present experiment was manipulated within-subjects ensures that whatever visual eccentricities might have been present for any particular participant, those eccentricities were present during each of the 18 combinations of the independent variables.

Another limitation of this experiment was inattentive participation in the online format. Failing the comprehension questionnaire disqualified 67 of the initial 140 participant sample. An exclusion rate as high as $47.9 \%$ is hypothesized to be due to a combination of participant inattentiveness in the online format and the strict inclusion criteria used (must score $100 \%$ on the quiz to be qualified). The success of future webbased educational interventions will rely on addressing this issue. Concise task instructions coupled with engaging activities and videos may be essential to fully engage and maintain participant attention.

Another limitation is evident by the greater variability in brightness predictions for the specular, 2-degree condition within the pre-demonstration $(M=0.098, \mathrm{SD}=$ $10.371)$ and within the demonstration $(M=6.876, \mathrm{SD}=9.023)$. This may indicate that participants had difficulty deciding where on the material sample to base their magnitude estimates. If future studies are to use specular samples as a comparison, it may be 
beneficial to add instructions that more clearly state how participants should rate these samples.

The tightly controlled conditions in which materials were presented also may have been at the sacrifice of generalizability to applied roadway environments. In particular, the results of the visual demonstration indicated that retroreflective materials received ratings that were significantly higher in the condition with the most extreme entrance angle $\left(45^{\circ}\right)$. Beaded retroreflective materials used on the roadway often experience degradation at or before the 45-degree entrance angle. This perplexing finding is primarily attributed to the tightly controlled and unnatural conditions under which the materials were displayed in this study. Instead of being affixed to a flat, rigid backing, like in this experiment, beaded retroreflective materials are normally attached to a garment and curved around the contours of a pedestrian. It is important that the results of this study and the exceptional performance of the retroreflective material used is understood as being within ideal conditions. Future research might be able to apply the results of this study in a way that is more directly applicable to pedestrian conspicuity in natural road environments.

The comparison of Szubski et al.'s magnitude estimates to the current study's magnitude estimates reveals another important limitation. While online visual demonstrations of retroreflection have shown to be effective in decreasing participants' estimates of brightness for diffuse and specular reflectors, it was not as effective at increasing retroreflective estimates as an in-person demonstration was (Figure 14). The primary benefit of an online demonstration, therefore, is the potential to increase the 
usage of retroreflective markings by emphasizing the lack of conspicuity diffuse clothing provides to vulnerable road users. It's possible that the difference in retroreflective brightness estimates between the two studies was not primarily caused by the presentation mode, but it is an important consideration for the future of educational interventions. Before deciding whether to utilize an in-person or online demonstration of retroreflection, the feasibility of each approach should be evaluated to determine which format would best increase a pedestrian's awareness of their own visibility in the nighttime roadway environment.

\section{Conclusions}

Even though road users frequently encounter retroreflective surfaces when driving at night, evidence continues to support that typical road users are unfamiliar with the principle of retroreflectivity. Because retroreflective materials do not look "special" when viewed under room illumination, typical road users may not appreciate the safety benefits that they can provide at night. And when they see retroreflective surfaces illuminated by their own headlamps it is usually from a distance that prevents close examination. It is our hope that this study represents the start of an evidence-based attempt to encourage the use of retroreflective markings by pedestrians and bicyclists at night. The goal of this study was to use participants' brightness judgments as a way of exploring road users' understanding (and misunderstanding) of the principle of retroreflectivity. The results of this study align with the idea that a simple visual demonstration - coupled with a succinct explanation - can help road users appreciate the principle of retroreflection in a way that might increase the usage of retroreflective markings by vulnerable road users. By 
conducting research to gauge road users' appreciation for retroreflective material's robustness to variations in entrance angles, we are better informed about misconceptions surrounding retroreflection, and better positioned to design effective educational interventions. 


\section{APPENDIX A}

\section{Demographic Questions}

How old are you?

In what college is your primary major?

$\square$ Agriculture, Forestry and Life Sciences

$\square$ Architecture, Arts and Humanities

$\square$ Behavioral, Social and Health Sciences

$\square$ Business

$\square$ Education

$\square$ Engineering, Computing and Applied Sciences

$\square$ Science (Astronomy, Biology, Chemistry, Statistics)

$\square$ Other

Do you currently have a valid driver's license?

$\square$ Yes

$\square$ No

How many years have you been a licensed driver?

$\square$ Less than 1 year

$\square 1$ year

$\square 2$ years

$\square 3$ years

$\square 4$ years

$\square 5$ or more years

Do you wear corrective lenses, such as eyeglasses or contact lenses?

$\square$ Yes

$\square$ No

Other than nearsightedness, farsightedness, or astigmatism, do you have any medical problems that prevent you from seeing well?

$\square$ Yes

$\square$ No 


\title{
APPENDIX B
}

\author{
Instructions for the Audio Check
}

Please turn on the sound on your device and press the "play" button below. Follow the audio instructions and type your unique password in the text box.

Instructions via sound clip:

"Adjust the volume on your device so that you hear my voice at a comfortable volume. You will need to leave the sound on throughout the experiment. If you are ready to continue the experiment, please type the code ' 152038 ' into the text box on your screen. Please use numbers only, no letters or spaces. Once again, your unique code is ' 1520 38." 


\section{APPENDIX C}

\section{Instructional Video Script}

"Our research group studies how well drivers see pedestrians and bicyclists at night, and we are looking for ways to make pedestrians and bicyclists more visible at night. In this experiment, we are studying how bright small squares made from different types of materials appear when they are illuminated at night. There are no tricks and no deception in this experiment.

This is a two-part experiment. First, you will be viewing video recordings of different squares. This is an example of a square that you will see in the videos. This is the camera that recorded the videos. This is a lamp that is always attached to the camera. Think of this lamp as being sort of like car headlights; they light up the area directly ahead. The lamp is either on or off. This is how it looks when the lamp is on; its intensity was never changed. The lamp was turned off during the recording of the videos in the first part of the experiment, but the lamp was turned on for the videos in the second half of the experiment.

Let me first describe part one of the experiment. During this phase, the room lights will be on and the lamp will be off. In Part 1, you will make predictions for how bright the squares will look when you later see them in part 2. When you make these predictions, I'd like you to imagine that the room lights are off and that the only light in the room is the light from this lamp

During part two, the room lights will be turned off, but the lamp will be turned on and it will be illuminating the squares.

In the videos, squares will slide in and out of the video frame, like this. Squares will also be displayed at different angles relative to the camera." 


\section{APPENDIX D}

Images from the Instructional Video

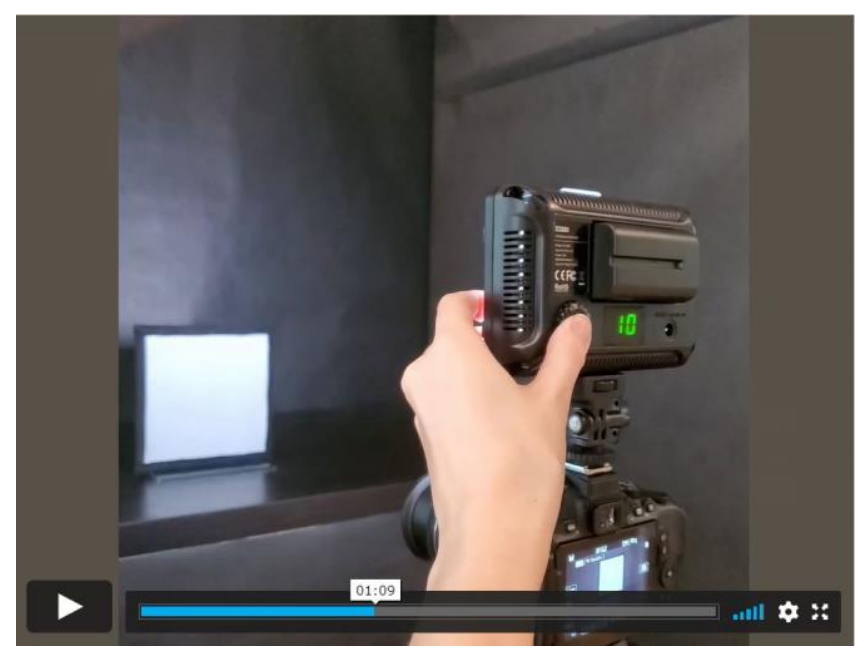

PART 1

Room lights: $O N$

b Lamp: OFF

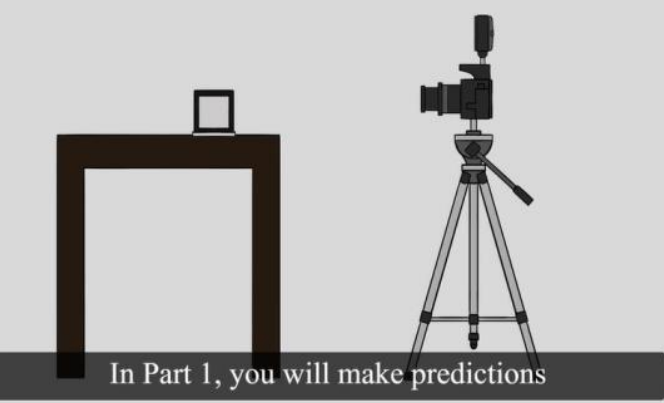

PART 1

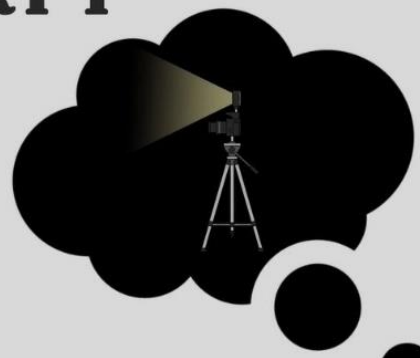

is the light from this lamp.

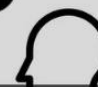



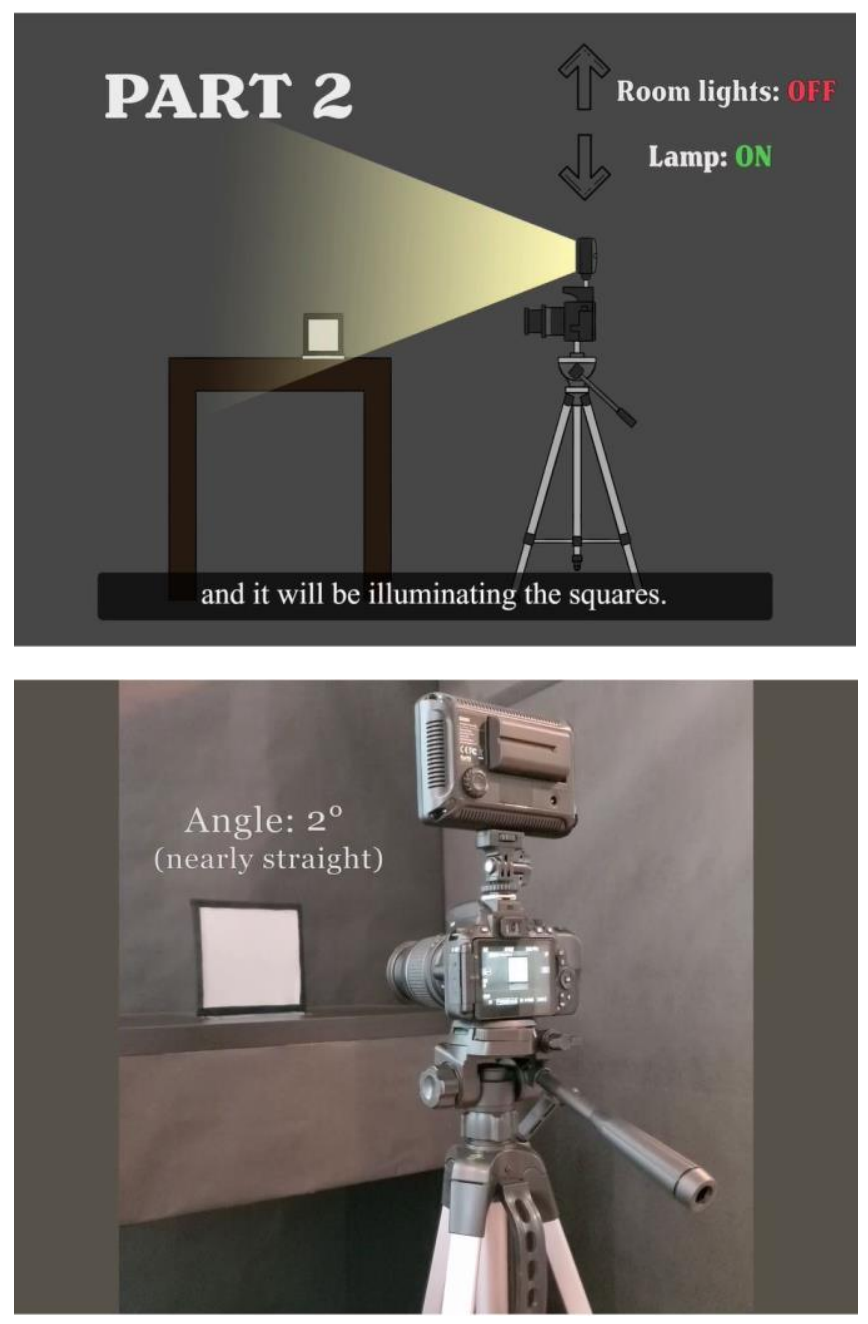


\section{APPENDIX E}

\section{Task Comprehension Questions}

1. In Part 1, you will be how bright the squares look
A. judging; right now
B. predicting; during Part 2

2. In Part 2, the room lights will be and the lamp will be
A. off; off
B. on; on
C. off; on
D. on; off

3. "200" is a valid answer.

$\square$ True

$\square$ False

4. ".05" is a valid answer.

$\square$ True

$\square$ False

5. " $-10 "$ is a valid answer.

$\square$ True

$\square$ False

6. " 0 " is a valid answer.

$\square$ True

$\square$ False

7. If the second video seems $5 x$ brighter than the first, what should your $2^{\text {nd }}$ value be?

8. Do you understand that any number greater than zero is valid? 


\section{APPENDIX F}

\section{Magnitude Estimation Task}

Part 1 (Pre-demo) task and video stimuli

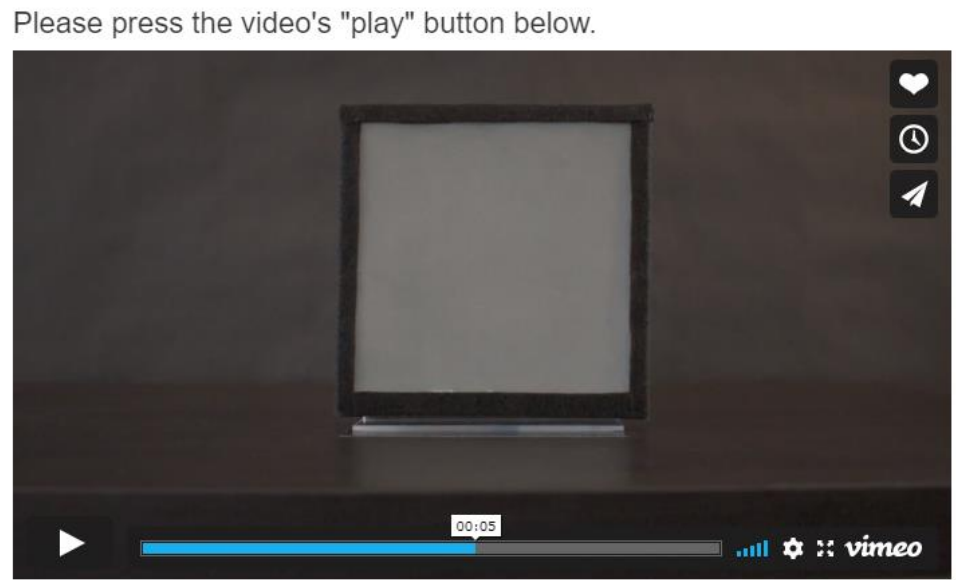

How bright do you predict this square will look when the room lights are turned off and the lamp is the only source of light in the room?

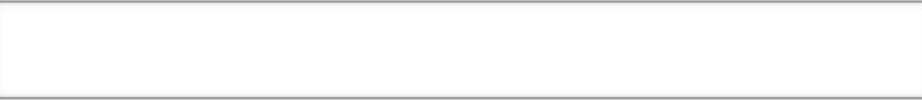

Part 2 (Demo) task and video stimuli

Please press the video's "play" button below.

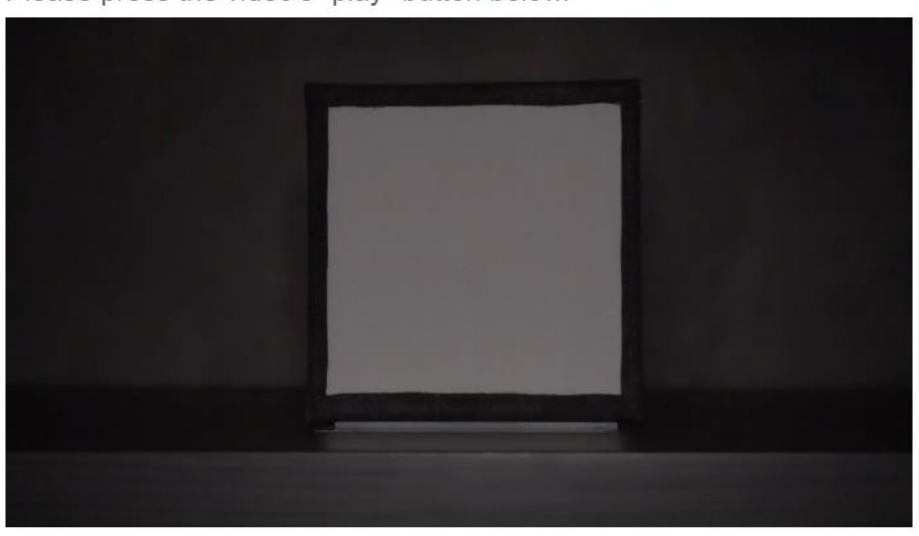

Now that the square is illuminated by the lamp, how bright does it look?

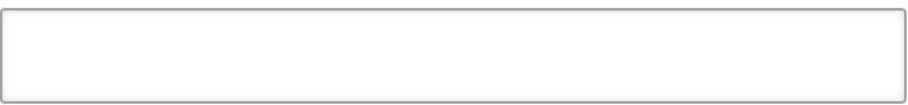




\section{APPENDIX G}

\section{Post-experiment Survey Questions}

1. Do you feel that you understood what you were supposed to do in this experiment? (You will earn your research credits regardless of how you answer this question.)

\section{$\square$ Yes}

$\square$ No

2. Please explain what part you might not have understood completely.

3. If you are walking near traffic during the day, what should you wear or use to make yourself most visible to an approaching driver?

a. White clothing

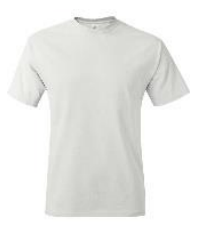

b. This vest

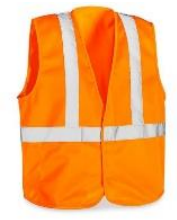

c. Florescent clothing

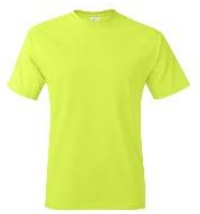

d. Flashlight

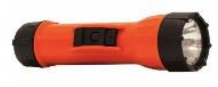

4. If you are walking near traffic at night, what should you wear or use to make yourself most visible to an approaching driver? 
a. White clothing

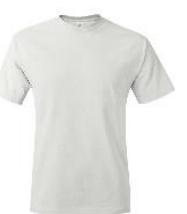

b. This vest

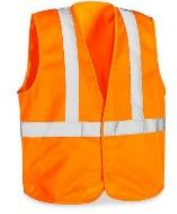

c. Florescent clothing

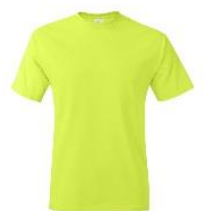

d. Flashlight

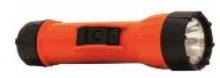

5. Are you familiar with the term "retroreflective?"

$\square$ Yes

$\square$ No

6. How is the meaning of "retroreflective" different from "reflective"? 


\section{REFERENCES}

Arecchi, A. V., Messadi, T., \& Koshel, R. J. (2007). Field Guide to Illumination. SPIE Press, 31-32.

Balk, S. A., Brooks, J. O., Klein, N., \& Grygier, J. (2012). Pedestrians' estimates of their own visibility: A simple and effective computer-based technique, Journal of Safety Research, 43(2), 101-106, ISSN 0022-4375. https://doi.org/10.1016/j.jsr.2012.01.002

Borzendowski, S. A. W., Sewall, A. A. S., Fekety, D. K., \& Tyrrell, R. A. (2014, September). Effects of an Educational Intervention on Athletes' Attitudes Toward Wearing Conspicuity-Enhancing Garments at Night. Proceedings of the Human Factors and Ergonomics Society Annual Meeting, 58(1), 2141-2145. Los Angeles, CA: Sage Publications.

Cohen, J. (1988). Statistical Power Analysis for the Behavioral Sciences (2nd ed.). Hillsdale, NJ: Lawrence Erlbaum Associates, Publishers.

Federal Highway Administration. (2001). Guidelines and Recommendations to Accommodate Older Drivers and Pedestrians (FHWA Publication No. 01-051). U.S. Department of Transportation. https://www.fhwa.dot.gov/publications/research/safety/humanfac/01051/01-051.pdf

Gravings, J. S., Tyrrell, R. A., \& Balk, S. A. (2009). Quantifying the Subjective Brightness of Retroreflective Material Using Magnitude Estimations. Proceedings of the Fifth International Driving Symposium on Human Factors in Driver Assessment, Training and Vehicle Design. Big Sky, Montana. 
Hagel, B. E., Lamy, A., Rizkallah, J. W., Belton, K. L., Jhangri, G. S., Cherry, N., et al. (2007). The prevalence and reliability of visibility aid and other risk factor data for uninjured cyclists and pedestrians in Edmonton, Alberta, Canada. Accident Analysis \& Prevention, 39, 284-289.

Marks, L. E. (1974). Sensory Processes: The New Psychophysics. New York: Academic Press.

Moskowitz, H.R. (1977). Magnitude Estimation: Notes on What, How, When, and Why to Use It. Journal of Food Quality, 1: 195-227. https://doi.org/10.1111/j.17454557.1977.tb00942.x

Retting, R. (2019). Pedestrian Traffic Fatalities by State: 2019 Preliminary Data. https://www.ghsa.org/sites/default/files/2020-02/GHSA-Pedestrian-SpotlightFINAL-rev2.pdf

Szubski, E. C., Fekety, D. K., Edewaard, D. E., \& Tyrrell, R. A. (2017). Perceptual judgements of the efficacy of visibility aids. Proceedings of the International Annual Meeting of the Human Factors and Ergonomics Society. Austin, Texas.

3M Occupational Health and Environmental Safety Division (2005). Retroreflection: Informed choices for enhanced visibility: the science, the performance, the product options [Brochure]. St. Paul, MN: n.p.

Teghtsoonian, M., \& Teghtsoonian, R. (1983). Consistency of individual exponents in crossmodal matching. Perception and Psychophysics. 33(3). 203-214. 
Tyrrell, R. A., Patton, C. W., \& Brooks, J. O. (2004). Educational interventions successfully reduce pedestrians' overestimates of their own nighttime visibility. Human Factors, 46(1), 170-182.

Tyrrell, R. A., Wood, J. M., Owens, D. A., Whetsel Borzendowski, S., \& Stafford Sewall, A. (2016). The conspicuity of pedestrians at night: A review. Clinical and experimental optometry, 99(5), 425-434.

Uding, K. D. (1993). Exact road geometry output program for retroreflective road sign performance. Transportation Research Board, Report No. 1421, 61-68.

Wolfe, J. M., Kluender, K. R., Levi, D. M., Bartoshuk, L. M., Herz, R. S., Klatzky, R. L., \& Merfeld, D. M. (2018). Sensation \& Perception (5th edition). NY: Sinauer Associates. ISBN: 9781605356419.

Wood, J. M., Lacherez, P. F., Marszalek, R. P., \& King, M. J. (2009). Drivers' and cyclists' experiences of sharing the road: Incidents, attitudes and perceptions of visibility. Accident Analysis \& Prevention, 41(4), 772-776. 